\title{
Proprioceptive Training for Knee Osteoarthritis: A Systematic Review and Meta-Analysis of Randomized Controlled Trials
}

\author{
Yi Wang ${ }^{1 \dagger}$, Zugui $\mathrm{Wu}^{1 \dagger}$, Zehua Chen ${ }^{1 \dagger}$, Xiangling Ye ${ }^{1 \dagger}$, Guoqian $\mathrm{Chen}^{2}$, Jiaman Yang ${ }^{1}$, \\ Peiming Zhang ${ }^{3}$, Fang Xie ${ }^{4}$, Yingxin Guan ${ }^{1}$, Jiatao Wu ${ }^{1}$, Weijian Chen ${ }^{1}$, Zixuan $\mathrm{Ye}^{1}$ and \\ Xuemeng $\mathrm{Xu}^{1,5 *}$ \\ ${ }^{1}$ The Fifth Clinical Medical College of Guangzhou University of Chinese Medicine, Guangzhou, China, ${ }^{2}$ Department of \\ Orthopaedic Surgery, Zhejiang Provincial Hospital of Chinese Medicine, Hangzhou, China, ${ }^{3}$ Medical College of Acu-Moxi and \\ Rehabilitation, Guangzhou University of Chinese Medicine, Guangzhou, China, ${ }^{4}$ Affiliated Changde Hospital, Hunan \\ University of Traditional Chinese Medicine, Changde, China, ${ }^{5}$ Guangdong Second Traditional Chinese Medicine Hospital, \\ Guangzhou, China
}

Background: There is increased interest in proprioceptive training for knee osteoarthritis (KOA). However, little consensus supports the effectiveness of this intervention.

\section{OPEN ACCESS}

Edited by:

Andrea P. Rossi,

Integrated University Hospital

Verona, Italy

Reviewed by:

Renato Gorga Bandeira De Mello, Federal University of Rio Grande do

Sul, Brazil

Valentina Muollo,

University of Verona, Italy

*Correspondence:

Xuemeng $X_{u}$

xuxuemeng@163.com

†These authors have contributed equally to this work

Specialty section: This article was submitted to Geriatric Medicine, a section of the journal

Frontiers in Medicine

Received: 24 April 2021 Accepted: 04 October 2021 Published: 28 October 2021

Citation

Wang Y, Wu Z, Chen Z, Ye X, Chen G, Yang J, Zhang P, Xie F, Guan Y, Wu J,

Chen W, Ye Z and Xu X (2021)

Proprioceptive Training for Knee Osteoarthritis: A Systematic Review and Meta-Analysis of Randomized Controlled Trials.

Front. Med. 8:699921. doi: 10.3389/fmed.2021.699921
Objective: This meta-analysis aimed to assess the effects of proprioceptive training on symptoms, function, and proprioception in people with KOA.

Methods: The PubMed, Cochrane Library, Web of Science, and EMBASE databases were systematically searched from the inception dates to April 16, 2021 for relevant randomized controlled trials (RCTs). Data were pooled by calculating the standardized mean differences (SMDs) and 95\% confidence intervals (Cls). A random-effects model was used for the analyses.

Results: A total of 24 RCTs involving 1,275 participants were included in our analysis. This study indicated that compared to no intervention, proprioceptive training significantly improved pain, stiffness, physical function, joint position sense (JPS), muscle strength, mobility, and knee ROM $(P<0.05)$ in people with KOA. When compared to other non-proprioceptive training, proprioceptive training provided better results in terms of JPS (SMD $\left.=-1.28,95 \% \mathrm{Cl}:[-1.64,-0.92], R^{2}=0 \%, P<0.00001\right)$ and mobility (timed walk over spongy surface) (SMD $=-0.76,95 \% \mathrm{Cl}$ : $[-1.33,-0.18], l^{2}=64 \%$, $P=0.01)$, and other results are similar. When proprioceptive training plus other nonproprioceptive training compared to other non-proprioceptive training, the two groups showed similar outcomes, but there was a greater improvement for JPS (SMD $=-1.54$, 95\%Cl: $\left.[-2.74,-0.34], I^{2}=79 \%, P=0.01\right)$, physical function $(\mathrm{SMD}=-0.34,95 \% \mathrm{Cl}$ : $\left.[-0.56,-0.12], I^{2}=0 \%, P=0.003\right)$, and knee ROM $(P<0.05)$ in the proprioceptive training plus other non-proprioceptive training group. When proprioceptive training plus conventional physiotherapy compared against conventional physiotherapy, the two groups demonstrated similar outcomes, but there was a significant improvement for JPS (SMD $=-0.95,95 \% \mathrm{Cl}$ : $\left.[-1.73,-0.18], I^{2}=78 \%, P=0.02\right)$ in the proprioceptive training plus conventional physiotherapy group.

Conclusions: Proprioceptive training is safe and effective in treating KOA. There is some evidence that proprioceptive training combined with general non-proprioceptive training 
or conventional physiotherapy appears to be more effective and should be considered as part of the rehabilitation program. However, given that the majority of current studies investigated the short-term effect of these proprioceptive training programs, more large-scale and well-designed studies with long-term follow up are needed to determine the long-term effects of these proprioceptive training regimes in KOA.

\section{Systematic Review Registration: https:/www.crd.york.ac.uk/prospero/\# recordDetails, PROSPERO, identifier: CRD42021240587.}

Keywords: proprioceptive training, knee osteoarthritis, rehabilitation, systematic review, meta-analysis

\section{INTRODUCTION}

Osteoarthritis (OA) is a chronic, degenerative joint disease that mainly affects weight-bearing joints (1). Knee osteoarthritis (KOA) is the most common form of $\mathrm{OA}$ and affects $\sim 265$ million people worldwide (2). Additionally, it is one of the leading causes of disability (3). Due to population aging and the increasing incidence of obesity, the prevalence of $\mathrm{KOA}$ is rising (4), increasing the socioeconomic burden for affected individuals and healthcare systems $(5,6)$. KOA is clinically characterized by pain, joint stiffness, reduced joint motion, quadriceps weakness, and proprioceptive deficits $(7,8)$. Its pathology may be associated with degenerative lesions in cartilage secondary to inflammation linked to hyperplasia and chondrocyte apoptosis $(9,10)$. The treatment options for knee osteoarthritis include non-pharmacological, pharmacological, or surgical measures $(11,12)$. Current clinical guidelines recommend a multimodal, individualized non-pharmacological treatment program as firstline treatment for $\operatorname{KOA}(13,14)$. The core treatment included therapeutic exercise (e.g., aerobic, resistance, strengthening, and proprioception), physical therapy (e.g., massage, ultrasound, and thermotherapy), lifestyle modifications, weight management, and education $(11,13,15-17)$. These interventions aimed to relieve pain, improve physical function, and slow the progression of the disease (18-20).

Although the etiology of KOA remains largely undefined (21), some risk factors that have been proved to influence KOA susceptibility include age, sex, obesity, knee injury, muscle weakness, genetics, and ethnicity (22-26). Furthermore, many recent studies indicated that proprioceptive impairments could be an important risk factor for the incidence and progression of KOA (27-31). Proprioception mostly consists of several different biomechanical components, such as JPS, motion sense, velocity, and force (32). Proprioception derives from proprioceptors in skeletal muscles, tendons, ligaments, and joint capsule (33-35). With the progression of KOA, proprioception could also be decreased $(30,36)$. Additionally, proprioceptive impairments could be a cause of knee pain or activity limitations in KOA patients $(29,37)$.

The programs focused on improving or restoring proprioception have been referred to as proprioceptive trainings (38). Recently, although many studies have explored and analyzed the efficacy of proprioceptive training for KOA
(39-41), several investigators have reported that there was insufficient clinical evidence of proprioceptive training for $\mathrm{KOA}$, and the results of previous studies have been also inconsistent $(35,42)$. To our knowledge, two previous reviews have reported on the effects of proprioceptive training in KOA $(35,43)$. However, one of these is now about 10 years old, and the number of studies included is small (35), whereas the one recent review did not compare the efficacy of proprioceptive training with other general non-proprioceptive training for KOA (42). Besides, these two reviews did not assess the safety of proprioceptive training and investigate the effects of combinations of proprioceptive training with other interventions in KOA. Our interest in updating the current evidence has increased as the numerous renewals of high-quality studies on proprioceptive training in treating KOA (43-45). Therefore, we conducted this study to summarize all current high-quality evidence on the clinical efficacy and safety of proprioceptive training for $\mathrm{KOA}$, and to provide a quantitative assessment. This will be very important and necessary, and the results of the study will provide evidence and guidance for the promotion and application of proprioceptive training in clinical practice.

\section{METHODS}

Methods and reporting of this systematic review and metaanalysis adhere to the Preferred Reporting Items for Systematic Reviews and Meta-Analyses (PRISMA) guidelines (46). The protocol for this meta-analysis was registered with PROSPERO (CRD 42021240587).

\section{Search Strategy}

We systematically searched the PubMed, Cochrane Library, Web of Science, and EMBASE databases from the inception dates to April, 16 2021. The following search string and $\mathrm{MeSH}$ terms were used to search: "Proprioceptive training," "Knee osteoarthritis," and "Randomized Controlled Trial." In addition, we also manually searched the reference lists of selected articles and reviews for additional relevant articles. Two independent reviewers (YW and ZW) screened eligible articles, all disagreements were resolved by independent thirdparty review and consensus. The search strategy is detailed in Supplementary Table S1. 


\section{Selection Criteria}

We developed eligibility criteria for this study using the (PICOS) description model (47) of participants, interventions, comparisons, outcomes, and study design.

\section{Participants}

Inclusion criteria:

- Adult patients with KOA

Exclusion criteria:

- Participants who had suffered knee joint trauma or surgery

- Participants with other muscular, joint, or neurological conditions.

\section{Intervention}

- Proprioceptive training

Proprioceptive training includes proprioceptive, balance, and sensorimotor training. However, no restrictions were made in terms of the frequency, duration, or intensity of the intervention. Additionally, we excluded studies where the intervention was whole-body vibration or water training.

\section{Comparators}

- Proprioceptive training vs. no intervention

- Proprioceptive training vs. other non-proprioceptive training (e.g., resistance and strength training)

- Proprioceptive training with other non-proprioceptive training vs. other non-proprioceptive training

- Proprioceptive training with conventional physiotherapy vs. conventional physiotherapy.

\section{Outcomes}

Primary outcomes:

- Pain [visual analog scale (VAS), numeric rating scale (NRS), Western Ontario and McMaster Universities Osteoarthritis Index (WOMAC) scale, Knee Injury and Osteoarthritis Outcome Score (KOOS)]

- Stiffness (WOMAC, KOOS)

- Physical function (WOMAC, KOOS)

Secondary outcomes:

- Joint position sense (JPS)

- Muscle strength

- Mobility [walking-speed timed test (WST), Get up and go (GUG) test]

- Knee range of motion (ROM)

- Balance

- Adverse events.

\section{Study Design}

- Randomized controlled trials (RCTs)

- Published in English.

\section{Data Extraction}

Two independent reviewers (YW and ZW) used a standardized form extract the following information from each study in accordance with the pre-specified study protocol, including participant characteristics (e.g., age, gender, stage of KOA), study characteristics (e.g., lead author, publication year, country of origin, intervention frequency and duration, follow-up period), and main outcomes. Disagreements were resolved by discussion and consensus between the reviewers. If necessary, we will contact the corresponding authors to obtain the required information.

\section{Quality Assessment}

The methodological quality of each included studies was assessed independently by two reviewers (YW and ZW) using the Physiotherapy Evidence Database (PEDro) scale $(46,48)$. The PEDro scale (range, 0-10, with 10 indicating highest quality) is a reliable and valid appraisal tool to assess the quality of physiotherapy-based RCTs $(48,49)$. Furthermore, we also assessed the overall quality of the evidence for each outcome through the Grades of Recommendations, Assessment, Development, and Evaluation (GRADE) approach (47). Any inconsistency was resolved through independent third-party review and consensus.

\section{Statistical Analysis}

Data analyses were performed using Revman (version 5.3, Cochrane Collaboration) and Stata (version 13.0). We converted other forms of data (i.e., median, mean [95\%CI], standard error and interquartile range) to means (SDs) based on the Cochrane Handbook for Systematic Reviews of Interventions (50). The standard mean differences (SMDs) with 95\% confidence intervals (95\% CIs) for pooled data were calculated. We pooled the data using a random-effects model and examined statistical heterogeneity by calculating the $I^{2}$ statistic. An $I^{2}$ statistic $>50 \%$ was considered to be substantially heterogeneous $(50,51)$. We planned to perform subgroup analyses to identify potential determinants of efficacy. Sensitivity analysis was also used to explore potential sources of heterogeneity between studies and to assess whether the significant results were robust. Furthermore, we evaluated publication bias by examining funnel plots and statistical asymmetry tests (Begg's test and Egger's regression). $P<0.05$ was considered statistically significant.

\section{RESULTS}

\section{Study Selection}

We retrieved 539 potentially relevant records through electronic and manual searching. EndNote X9 (Bld 12062) was used to screen eligible studies. Initially, 388 articles remained after removal of duplicates. Then we selected 40 articles for fulltext review after screening their titles and abstracts. Fourteen articles were excluded since they did not meet inclusion criteria (e.g., non-randomization, no relevant outcome, ineligible intervention, non-English); two studies did not provide complete data for calculation of effect sizes. Ultimately, we selected 24 studies (39-41, 43-45, 52-69) for inclusion in our study (Figure 1). 


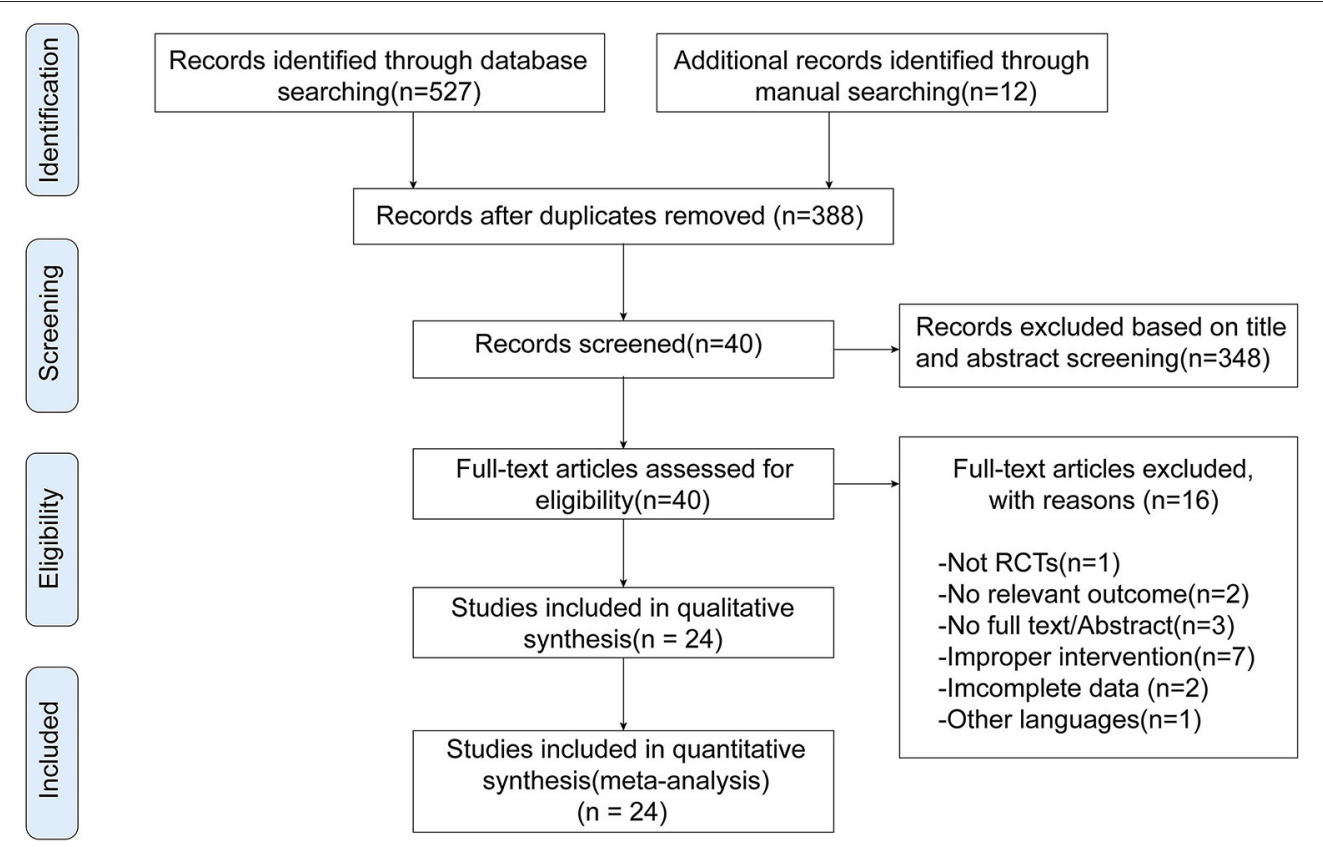

FIGURE 1 | Flowchart of study selection.

\section{Study Characteristics}

Twenty-four studies involving a total of 1,275 participants with KOA were included. The studies were from Brazil (52), Turkey (60, 64, 66, 69), Australia (58), Canada (59), Thailand (62), Egypt (55, 65), Iran (44), Malaysia (63), the United States (40, 41, 61), India (39, 43, 54, 68), and China (45, 53, 56, $57,67)$. The publication intervals of 24 included articles were from 2005 to 2020, and the sample size ranging from 15 to 183 participants. Twenty-two studies reported the age of the participants. Their mean (SD) age ranged from 40.87 (4.91) to 72.4 (11.02) years. Except for three studies $(39,54$, 61) that did not specify diagnostic criteria, all other studies used the Kellgren and Lawrence (70) radiological assessment scale or the American College of Rheumatologist's diagnostic classification (71) to diagnose KOA. Regarding the comparison of interventions, seven studies compared proprioceptive training to no intervention $(40,45,56,57,59,60,67)$, eight studies compared proprioceptive training to other non-proprioceptive training $(40,52,56,57,61,62,67,68)$, seven studies compared proprioceptive training plus other non-proprioceptive training to other non-proprioceptive training $(40,41,55,58,64-$ 66 ), and seven studies compared proprioceptive training plus conventional physiotherapy to conventional physiotherapy (39, $43,44,53,54,63,69)$.

The duration of the intervention ranges from 2 to 16 weeks. In addition, the shortest follow-up period was only 2 weeks (63) and the longest was 52 weeks $(41,66)$. The characteristics of the included studies are summarized in Table $\mathbf{1 .}$ And the detailed descriptions of interventions are presented in Supplementary Table S2.

\section{Quality of Studies}

The mean PEDro scale score for all studies was 6.25 (range, 4-8; Table 2), suggesting that the studies were of moderate quality. All 24 studies satisfied four of the PEDro criteria, namely "random allocation," "similar baseline," "between-group statistics," and "point measures," but only eight studies (40, 41, 52, 57-59, 61, 62) used concealed allocation to minimize allocation bias. However, except for two $(29,38)$, the remaining studies did not account for "the blinding of the subjects and therapists," $12(39,41,44,52,53$, $55,57-59,64,66,67)$ of the studies employed assessor blinding. In addition, six of the studies $(40,41,43,45,53,61)$ lost more than $15 \%$ participants during follow-up and the inconsistent use of "intention-to-treat" analyses were found to be consistent trial limitations in most of the studies.

\section{Assessed Outcomes and Evidence Synthesis Primary Outcomes \\ Pain}

Nine studies $(40,44,45,53,57,58,61,66,69)$ used the WOMAC pain subscale to assess pain. Subgroup analysis showed significant improvement in pain for proprioceptive training compared to no intervention (SMD $=-1.07,95 \% \mathrm{CI}$ : $[-1.46,-0.68], I^{2}$ $=0 \%, P<0.00001)$. When proprioceptive training compared against other non-proprioceptive training, the meta-analysis showed no statistically significant difference in alleviating pain $\left(\mathrm{SMD}=-0.02,95 \% \mathrm{CI}\right.$ : $\left.[-0.74,0.69], I^{2}=0 \%, P=0.95\right)$. When proprioceptive training plus other non-proprioceptive training compared to other non-proprioceptive training, the meta-analysis revealed no significant difference $(\mathrm{SMD}=-0.17$, 
TABLE 1 | Characteristics of the included studies.

\begin{tabular}{|c|c|c|c|c|c|c|c|}
\hline $\begin{array}{l}\text { References: publication } \\
\text { year, country }\end{array}$ & $\begin{array}{l}\text { Study } \\
\text { design }\end{array}$ & Participant characteristics & $\begin{array}{l}\text { Diagnosis } \\
\text { criteria/K-L } \\
\text { grade }\end{array}$ & Intervention & $\begin{array}{l}\text { Intervention } \\
\text { characteristics, } \\
\text { follow-up }\end{array}$ & Outcome measures & PEDro score \\
\hline $\begin{array}{l}\text { Gomiero et al. (52), 2018, } \\
\text { Brazil }\end{array}$ & $\mathrm{RCT}$ & $\begin{array}{l}\text { No. (M/F): } 64(3 / 61) \\
\text { IG: } 32(2 / 30) \\
\text { CG: } 32(1 / 31) \\
\text { Dropout: } 2 \\
\text { Mean Age } \pm \text { SD, years: } \\
\text { IG: } 61.6 \pm 6.8 \\
\text { CG: } 61.8 \pm 6.4\end{array}$ & ACR/K-L I-IV & $\begin{array}{l}\text { IG: Proprioceptive training } \\
\text { CG: Resistance training }\end{array}$ & $\begin{array}{l}16 \text { weeks, } \\
2 \text { sessions/week }\end{array}$ & $\begin{array}{l}\text { VAS; } \\
\text { WOMAC (total); } \\
\text { Mobility; } \\
\text { Balance }\end{array}$ & 8 \\
\hline $\begin{array}{l}\text { Tsauo et al. (53), 2008, } \\
\text { Taiwan, China }\end{array}$ & $\mathrm{RCT}$ & $\begin{array}{l}\text { No. }(M / F): 29(5 / 24) \\
\text { IG: } 15(1 / 14) \\
\text { CG: } 14(4 / 10) \\
\text { Dropout: } 31 \\
\text { Mean Age } \pm \text { SD, years: } \\
\text { IG: } 61.7 \pm 6.6 \\
\text { CG: } 60.1 \pm 6.7\end{array}$ & ACR/K-L II-III & $\begin{array}{l}\text { IG: Proprioceptive training plus } \\
\text { Conventional physiotherapy } \\
\text { CG: Conventional physiotherapy }\end{array}$ & $\begin{array}{l}8 \text { weeks, } \\
3 \text { sessions/week, } \\
30 \mathrm{~min} / \mathrm{session}\end{array}$ & $\begin{array}{l}\text { WOMAC (pain); } \\
\text { WOMAC (stiffness); } \\
\text { WOMAC (physical function); } \\
\text { JPS }\end{array}$ & 5 \\
\hline $\begin{array}{l}\text { Kumar et al. (54), 2013, } \\
\text { India }\end{array}$ & $\mathrm{RCT}$ & $\begin{array}{l}\text { No. }(M / F): 44(19 / 25) \\
\text { IG: } 22 \\
\text { CG: } 22 \\
\text { Dropout: } 0 \\
\text { Mean Age } \pm \text { SD, years: } \\
\text { IG: } 53.18 \pm 6.88 \\
\text { CG: } 53.32 \pm 5.36\end{array}$ & NA & $\begin{array}{l}\text { IG: Proprioceptive training plus } \\
\text { Conventional physiotherapy } \\
\text { CG: Conventional physiotherapy }\end{array}$ & $\begin{array}{l}4 \text { weeks, } \\
3 \text { sessions/week }\end{array}$ & $\begin{array}{l}\text { NRS; } \\
\text { WOMAC (physical function); } \\
\text { JPS }\end{array}$ & 6 \\
\hline $\begin{array}{l}\text { Fitzgerald et al. (41), 2011, } \\
\text { USA }\end{array}$ & $\mathrm{RCT}$ & $\begin{array}{l}\text { No. (M/F): } 183 \text { (61/122) } \\
\text { IG: } 91 \text { (31/60) } \\
\text { CG: } 92(30 / 62) \\
\text { Dropout: } 38 \\
\text { Mean Age } \pm \text { SD, years: } \\
\text { IG: } 63.3 \pm 8.9 \\
\text { CG: } 64.6 \pm 8.4\end{array}$ & ACR/K-L II-IV & $\begin{array}{l}\text { IG: Proprioceptive training plus } \\
\text { Strength training } \\
\text { CG: Strength training }\end{array}$ & $\begin{array}{l}8,26, \text { and } 52 \text { weeks, } \\
3 \text { sessions/week, } \\
\geq 30 \mathrm{~min} / \text { session }\end{array}$ & $\begin{array}{l}\text { NRS; } \\
\text { WOMAC (physical function); } \\
\text { WOMAC (total); } \\
\text { Mobility }\end{array}$ & 7 \\
\hline $\begin{array}{l}\text { Rogers et al. (40), 2012, } \\
\text { USA }\end{array}$ & $\mathrm{RCT}$ & $\begin{array}{l}\text { No. }(M / F): 33(13 / 20) \\
\text { IG (PT): } 8 \\
\text { CG (RT): } 8 \\
\text { CG (PT + RT): } 9 \\
\text { CG: } 8 \\
\text { Dropout: } 11 \\
\text { Mean Age } \pm \text { SD, years: } \\
\text { IG (PT): } 70.7 \pm 10.7 \\
\text { CG (RT): } 70.8 \pm 6.5 \\
\text { CG (PT + RT): } 68.8 \pm 10.1 \\
\text { CG: } 71.2 \pm 10.9\end{array}$ & ACR & $\begin{array}{l}\text { IG (PT): Proprioceptive training } \\
\text { CG (RT): Resistance training } \\
\text { CG (PT + RT): Proprioceptive } \\
\text { training plus Resistance training } \\
\text { CG: Without intervention }\end{array}$ & $\begin{array}{l}8 \text { weeks, } \\
3 \text { sessions/week, } \\
30-40 \mathrm{~min} / \mathrm{session}\end{array}$ & $\begin{array}{l}\text { WOMAC (pain); } \\
\text { WOMAC (stiffness); } \\
\text { WOMAC (physical function); } \\
\text { WOMAC (total) }\end{array}$ & 6 \\
\hline
\end{tabular}


TABLE 1 | Continued

\begin{tabular}{|c|c|c|c|c|c|c|c|}
\hline $\begin{array}{l}\text { References: publication } \\
\text { year, country }\end{array}$ & $\begin{array}{l}\text { Study } \\
\text { design }\end{array}$ & Participant characteristics & $\begin{array}{l}\text { Diagnosis } \\
\text { criteria/K-L } \\
\text { grade }\end{array}$ & Intervention & $\begin{array}{l}\text { Intervention } \\
\text { characteristics, } \\
\text { follow-up }\end{array}$ & Outcome measures & PEDro score \\
\hline $\begin{array}{l}\text { Ahmed et al. (55), 2011, } \\
\text { Egypt }\end{array}$ & $\mathrm{RCT}$ & $\begin{array}{l}\text { No. }(M / F): 40 \\
\text { IG: } 20 \\
\text { CG: } 20 \\
\text { Dropout: } 0 \\
\text { Mean Age } \pm \text { SD, years: } \\
\text { IG: } 60 \pm 3.6 \\
\text { CG: } 62 \pm 3.2\end{array}$ & ACR/K-L II & $\begin{array}{l}\text { IG: Proprioceptive training plus } \\
\text { Traditional exercise } \\
\text { CG: Traditional exercise }\end{array}$ & $\begin{array}{l}6 \text { weeks, } \\
3 \text { sessions/week }\end{array}$ & $\begin{array}{l}\text { VAS; } \\
\text { JPS }\end{array}$ & 7 \\
\hline $\begin{array}{l}\text { Lin et al. (56), 2007, } \\
\text { Taiwan, China }\end{array}$ & $\mathrm{RCT}$ & $\begin{array}{l}\text { No. (M/F):81 (19/62) } \\
\text { IG (PT): 29(9/20) } \\
\text { CG (RT): 26(5/21) } \\
\text { CG: } 26(5 / 21) \\
\text { Dropout: } 8 \\
\text { Mean Age } \pm \text { SD, years: } \\
\text { IG (PT): } 61.6 \pm 8.1 \\
\text { CG (RT): } 61.0 \pm 7.7 \\
\text { CG: } 62.8 \pm 6.3\end{array}$ & ACR/K-L I-III & $\begin{array}{l}\text { IG (PT): Proprioceptive training } \\
\text { CG (RT): Resistance training } \\
\text { CG: Without intervention }\end{array}$ & $\begin{array}{l}8 \text { weeks, } \\
3 \text { sessions/week }\end{array}$ & Muscle strength & 5 \\
\hline $\begin{array}{l}\text { Jahanjoo et al. (44), 2019, } \\
\text { Iran }\end{array}$ & $\mathrm{RCT}$ & $\begin{array}{l}\text { No. (M/F):60 (13/47) } \\
\text { IG: 30(8/22) } \\
\text { CG: } 30(5 / 25) \\
\text { Dropout: } 0 \\
\text { Mean Age } \pm \text { SD, years: } \\
\text { IG: } 57.53 \pm 0.8 \\
\text { CG: } 55.57 \pm 1.6\end{array}$ & ACR/K-L I-III & $\begin{array}{l}\text { IG: Proprioceptive training plus } \\
\text { Conventional physiotherapy } \\
\text { CG: Conventional physiotherapy }\end{array}$ & $\begin{array}{l}5 \text { weeks, } \\
2 \text { sessions/week }\end{array}$ & $\begin{array}{l}\text { VAS; } \\
\text { WOMAC (pain); } \\
\text { WOMAC (stiffness); } \\
\text { WOMAC (physical function); } \\
\text { WOMAC (total); } \\
\text { Mobility }\end{array}$ & 7 \\
\hline $\begin{array}{l}\text { Lin et al. (57), 2009, } \\
\text { Taiwan, China }\end{array}$ & $\mathrm{RCT}$ & $\begin{array}{l}\text { No. (M/F): } 108(33 / 75) \\
\text { IG (PT): } 36(11 / 25) \\
\text { CG (ST): } 36(12 / 24) \\
\text { CG: } 36(10 / 26) \\
\text { Dropout: } 5 \\
\text { Mean Age } \pm \text { SD, years: } \\
\text { IG (PT): } 63.7 \pm 8.2 \\
\text { CG (ST): } 61.6 \pm 7.2 \\
\text { CG: } 62.2 \pm 6.7\end{array}$ & K-L I-IIII & $\begin{array}{l}\text { IG (PT): Proprioceptive training } \\
\text { CG (ST): Strength training } \\
\text { CG: Without intervention }\end{array}$ & $\begin{array}{l}8 \text { weeks, } \\
3 \text { sessions/week }\end{array}$ & $\begin{array}{l}\text { WOMAC (pain); } \\
\text { WOMAC (physical function); } \\
\text { JPS; } \\
\text { Mobility }\end{array}$ & 8 \\
\hline $\begin{array}{l}\text { Gohil and Shukla (43), } \\
\text { 2020, India }\end{array}$ & $\mathrm{RCT}$ & $\begin{array}{l}\text { No. }(M / F): 22 \\
\text { IG: } 11 \\
\text { CG:11 } \\
\text { Dropout: } 0\end{array}$ & K-L II-III & $\begin{array}{l}\text { IG: Proprioceptive training plus } \\
\text { Conventional physiotherapy } \\
\text { CG: Conventional physiotherapy }\end{array}$ & $\begin{array}{l}8 \text { weeks, } \\
3 \text { sessions/week }\end{array}$ & $\begin{array}{l}\text { NRS; } \\
\text { WOMAC (physical function); } \\
\text { JPS }\end{array}$ & 4 \\
\hline $\begin{array}{l}\text { Kirthika et al. (39), 2018, } \\
\text { India }\end{array}$ & $\mathrm{RCT}$ & $\begin{array}{l}\text { No. }(M / F): 40 \\
\text { IG: } 20 \\
\text { CG: } 20 \\
\text { Dropout: } 3 \\
\text { Mean Age } \pm \text { SD, years: } \\
\text { IG: } 58.2 \pm 5.7 \\
\text { CG: } 58.8 \pm 5.3\end{array}$ & NA & $\begin{array}{l}\text { IG: Proprioceptive training plus } \\
\text { Conventional physiotherapy } \\
\text { CG: Conventional physiotherapy }\end{array}$ & $\begin{array}{l}12 \text { weeks, } \\
5 \text { sessions/week }\end{array}$ & $\begin{array}{l}\text { VAS; } \\
\text { WOMAC (total) }\end{array}$ & 6 \\
\hline
\end{tabular}


TABLE 1 | Continued

\begin{tabular}{|c|c|c|c|c|c|c|c|}
\hline $\begin{array}{l}\text { References: publication } \\
\text { year, country }\end{array}$ & $\begin{array}{l}\text { Study } \\
\text { design }\end{array}$ & Participant characteristics & $\begin{array}{l}\text { Diagnosis } \\
\text { criteria/K-L } \\
\text { grade }\end{array}$ & Intervention & $\begin{array}{l}\text { Intervention } \\
\text { characteristics, } \\
\text { follow-up }\end{array}$ & Outcome measures & PEDro score \\
\hline $\begin{array}{l}\text { Pazit et al. (58), 2018, } \\
\text { Australia }\end{array}$ & $\mathrm{RCT}$ & $\begin{array}{l}\text { No. }(M / F): 19(9 / 10) \\
\text { IG: } 10(4 / 6) \\
\text { CG: } 9(5 / 4) \\
\text { Dropout: } 0 \\
\text { Mean Age } \pm \text { SD, years: } \\
\text { IG: } 65.10 \pm 4.77 \\
\text { CG: } 67.78 \pm 6.28\end{array}$ & ACR & $\begin{array}{l}\text { IG: Proprioceptive training plus } \\
\text { Resistance training } \\
\text { CG: Resistance training }\end{array}$ & $\begin{array}{l}8 \text { weeks, } \\
2 \text { sessions/week }\end{array}$ & $\begin{array}{l}\text { WOMAC (pain); } \\
\text { WOMAC (stiffness); } \\
\text { WOMAC (physical function); } \\
\text { WOMAC (total); } \\
\text { Mobility }\end{array}$ & 8 \\
\hline $\begin{array}{l}\text { Takacs et al. (59), } 2017 \text {, } \\
\text { Canada }\end{array}$ & $\mathrm{RCT}$ & $\begin{array}{l}\text { No. }(M / F): 36(6 / 30) \\
\text { IG: } 17(0 / 17) \\
\text { CG: } 19(6 / 13) \\
\text { Dropout: } 4 \\
\text { Mean Age } \pm \text { SD, years: } \\
\text { IG: } 66.1 \pm 8.7 \\
\text { CG: } 67.1 \pm 5.4\end{array}$ & ACR/K-L II-IV & $\begin{array}{l}\text { IG: Proprioceptive training } \\
\text { CG: Without intervention }\end{array}$ & $\begin{array}{l}10 \text { weeks, } \\
4 \text { sessions/week }\end{array}$ & $\begin{array}{l}\text { NRS; } \\
\text { WOMAC (physical function); } \\
\text { Muscle strength }\end{array}$ & 7 \\
\hline $\begin{array}{l}\text { Sekir and Gür (60), } \\
\text { 2005, Turkey }\end{array}$ & $\mathrm{RCT}$ & $\begin{array}{l}\text { No. (M/F):22(6/16) } \\
\text { IG: } 12(3 / 9) \\
\text { CG: } 10(3 / 7) \\
\text { Dropout: } 0 \\
\text { Mean Age } \pm \text { SD, years: } \\
\text { IG: } 59 \pm 8.9 \\
\text { CG: } 62 \pm 8.1\end{array}$ & ACR/K-L II-III & $\begin{array}{l}\text { IG: Proprioceptive training } \\
\text { CG: Without intervention }\end{array}$ & $\begin{array}{l}6 \text { weeks, } \\
2 \text { sessions/week }\end{array}$ & $\begin{array}{l}\text { VAS; } \\
\text { JPS; } \\
\text { Muscle strength }\end{array}$ & 6 \\
\hline $\begin{array}{l}\text { Rogers et al. (61), 2011, } \\
\text { USA }\end{array}$ & $\mathrm{RCT}$ & $\begin{array}{l}\text { No. }(M / F): 15 \\
\text { IG: } 6 \\
\text { CG: } 9 \\
\text { Dropout: } 5 \\
\text { Mean Age } \pm \text { SD, years: } \\
\text { IG: } 69.29 \pm 11.36 \\
\text { CG: } 72.4 \pm 11.02\end{array}$ & NA & $\begin{array}{l}\text { IG: Proprioceptive training } \\
\text { CG: Strength training }\end{array}$ & $\begin{array}{l}8 \text { weeks, } \\
3 \text { sessions/week, } \\
30 \mathrm{~min} / \text { session }\end{array}$ & $\begin{array}{l}\text { WOMAC (pain); } \\
\text { WOMAC (stiffness); } \\
\text { WOMAC (physical function); } \\
\text { Mobility }\end{array}$ & 5 \\
\hline $\begin{array}{l}\text { Chaipinyo and } \\
\text { Karoonsupcharoen (62), } \\
\text { 2009, Thailand }\end{array}$ & $\mathrm{RCT}$ & $\begin{array}{l}\text { No. (M/F):48(11/31) } \\
\text { IG: } 24(9 / 15) \\
\text { CG: } 24(2 / 22) \\
\text { Dropout: } 6 \\
\text { Mean Age } \pm \text { SD, years: } \\
\text { IG: } 62 \pm 6 \\
\text { CG: } 70 \pm 6\end{array}$ & ACR & $\begin{array}{l}\text { IG: Proprioceptive training } \\
\text { CG: Strength training }\end{array}$ & $\begin{array}{l}4 \text { weeks, } \\
5 \text { sessions/week }\end{array}$ & $\begin{array}{l}\text { KOOS (pain); } \\
\text { KOOS (symptoms); } \\
\text { Muscle strength; } \\
\text { Mobility }\end{array}$ & 6 \\
\hline $\begin{array}{l}\text { Mondam et al. (63), 2012, } \\
\text { Malaysia }\end{array}$ & $\mathrm{RCT}$ & $\begin{array}{l}\text { No. }(M / F): 50 \\
\text { IG: } 25 \\
\text { CG: } 25 \\
\text { Dropout: } 0\end{array}$ & K-L I-II & $\begin{array}{l}\text { IG: Proprioceptive training plus } \\
\text { Conventional physiotherapy } \\
\text { CG: Conventional physiotherapy }\end{array}$ & $\begin{array}{l}2 \text { weeks, } \\
7 \text { sessions/week }\end{array}$ & $\begin{array}{l}\text { VAS; } \\
\text { WOMAC (total); } \\
\text { Knee ROM }\end{array}$ & 6 \\
\hline $\begin{array}{l}\text { Diracoglu et al. (64), 2005, } \\
\text { Turkey }\end{array}$ & $\mathrm{RCT}$ & $\begin{array}{l}\text { No. }(M / F): 66(0 / 66) \\
\text { IG: } 32 \\
\text { CG: } 28 \\
\text { Dropout: } 6 \\
\text { Mean Age } \pm \text { SD, years: } \\
\text { IG: } 50.3 \pm 6.5 \\
\text { CG: } 50.8 \pm 7.9\end{array}$ & ACR/K-L I-II & $\begin{array}{l}\text { IG: Proprioceptive training plus } \\
\text { Strength training } \\
\text { CG: Strength training }\end{array}$ & $\begin{array}{l}8 \text { weeks, } \\
3 \text { sessions/week }\end{array}$ & WOMAC (physical function) & 6 \\
\hline
\end{tabular}


TABLE 1 | Continued

\begin{tabular}{|c|c|c|c|c|c|c|c|}
\hline $\begin{array}{l}\text { References: publication } \\
\text { year, country }\end{array}$ & $\begin{array}{l}\text { Study } \\
\text { design }\end{array}$ & Participant characteristics & $\begin{array}{l}\text { Diagnosis } \\
\text { criteria/K-L } \\
\text { grade }\end{array}$ & Intervention & $\begin{array}{l}\text { Intervention } \\
\text { characteristics, } \\
\text { follow-up }\end{array}$ & Outcome measures & PEDro score \\
\hline $\begin{array}{l}\text { Elgendy et al. (65), 2005, } \\
\text { Egypt }\end{array}$ & $\mathrm{RCT}$ & $\begin{array}{l}\text { No. }(M / F): 30 \\
\text { IG: } 15 \\
\text { CG:15 } \\
\text { Dropout: } 0\end{array}$ & ACR & $\begin{array}{l}\text { IG: Proprioceptive training plus } \\
\text { Traditional exercise } \\
\text { CG: Traditional exercise }\end{array}$ & $\begin{array}{l}8 \text { weeks, } \\
3 \text { sessions/week }\end{array}$ & $\begin{array}{l}\text { VAS; } \\
\text { JPS }\end{array}$ & 6 \\
\hline $\begin{array}{l}\text { Song et al. (45), } \\
\text { 2020, China }\end{array}$ & $\mathrm{RCT}$ & $\begin{array}{l}\text { No. (M/F):29(11/18) } \\
\text { IG: } 13(5 / 8) \\
\text { CG: } 16(6 / 10) \\
\text { Dropout: } 7 \\
\text { Mean Age } \pm \text { SD, years: } \\
\text { IG: } 68.5 \pm 4.3 \\
\text { CG: } 67.4 \pm 3.4\end{array}$ & K-L I-IIII & $\begin{array}{l}\text { IG: Proprioceptive training } \\
\text { CG: Without intervention }\end{array}$ & $\begin{array}{l}12 \text { weeks, } \\
3 \text { sessions/week, } \\
60 \mathrm{~min} / \mathrm{session}\end{array}$ & $\begin{array}{l}\text { WOMAC (pain); } \\
\text { Knee ROM }\end{array}$ & 4 \\
\hline $\begin{array}{l}\text { Diracoglu et al. (66), 2008, } \\
\text { Turkey }\end{array}$ & RCT & $\begin{array}{l}\text { No. }(M / F): 66(0 / 66) \\
\text { IG: } 32 \\
\text { CG: } 28 \\
\text { Dropout: } 6 \\
\text { Mean Age } \pm \text { SD, years: } \\
\text { IG: } 50.3 \pm 6.5 \\
\text { CG: } 50.8 \pm 7.9\end{array}$ & ACR/K-L I-II & $\begin{array}{l}\text { IG: Proprioceptive training plus } \\
\text { Strength training } \\
\text { CG: Strength training }\end{array}$ & $\begin{array}{l}8.52 \text { weeks, } \\
3 \text { sessions/week }\end{array}$ & $\begin{array}{l}\text { WOMAC (pain); } \\
\text { WOMAC (stiffness); } \\
\text { WOMAC (physical function); } \\
\text { WOMAC (total) }\end{array}$ & 6 \\
\hline $\begin{array}{l}\text { Jan et al. (67), 2009, } \\
\text { Taiwan, China }\end{array}$ & $\mathrm{RCT}$ & $\begin{array}{l}\text { No. (M/F):106(0/66) } \\
\text { IG(PT): 36(12/24) } \\
\text { CG(RT): 35(10/25) } \\
\text { CG:35(11/24) } \\
\text { Dropout: } 6 \\
\text { Mean Age } \pm \text { SD, years: } \\
\text { IG(PT): } 62.0 \pm 6.7 \\
\text { CG(RT): } 63.2 \pm 6.8 \\
\text { CG:62.2 } \pm 6.7\end{array}$ & ACR/K-L I-III & $\begin{array}{l}\text { IG (PT): Proprioceptive training } \\
\text { CG (RT): Resistance training } \\
\text { CG: Without intervention }\end{array}$ & $\begin{array}{l}8 \text { weeks, } \\
3 \text { sessions/week }\end{array}$ & $\begin{array}{l}\text { WOMAC (physical function); } \\
\text { JPS; } \\
\text { Muscle strength; } \\
\text { Mobility }\end{array}$ & 7 \\
\hline $\begin{array}{l}\text { Vamsidhar et al. (68), } \\
\text { 2017, India }\end{array}$ & $\mathrm{RCT}$ & $\begin{array}{l}\text { No. }(\mathrm{M} / \mathrm{F}): 30 \\
\text { IG: } 15 \\
\text { CG: } 15 \\
\text { Dropout: } 0 \\
\text { Mean Age } \pm \text { SD, years: } \\
\text { IG: } 40.87 \pm 4.91 \\
\text { CG: } 41.53 \pm 6.28\end{array}$ & K-L II-III & $\begin{array}{l}\text { IG: Proprioceptive training } \\
\text { CG: Strength training }\end{array}$ & $\begin{array}{l}3 \text { weeks, } \\
3 \text { sessions/week }\end{array}$ & $\begin{array}{l}\text { VAS; } \\
\text { WOMAC (total) }\end{array}$ & 6 \\
\hline $\begin{array}{l}\text { Duman et al. (69), 2012, } \\
\text { Turkey }\end{array}$ & $\mathrm{RCT}$ & $\begin{array}{l}\text { No. (M/F): } 54(5 / 49) \\
\text { IG: } 30 \\
\text { CG: } 24 \\
\text { Dropout: } 0 \\
\text { Mean Age } \pm \text { SD, years: } \\
64 \pm 3.7\end{array}$ & ACR/K-L III-IV & $\begin{array}{l}\text { IG: Proprioceptive training plus } \\
\text { Conventional physiotherapy plus } \\
\text { NSAID meloxicam } \\
\text { CG: Conventional physiotherapy } \\
\text { plus NSAID meloxicam }\end{array}$ & $\begin{array}{l}3 \text { weeks, } \\
5 \text { sessions/week }\end{array}$ & $\begin{array}{l}\text { WOMAC (pain); } \\
\text { WOMAC (stiffness); } \\
\text { WOMAC (physical function); } \\
\text { WOMAC (total); } \\
\text { JPS }\end{array}$ & 6 \\
\hline
\end{tabular}

ACR, American College of Rheumatologists; CG, control group; CP, conventional physiotherapy; F, female; IG, intervention group; JPS, joint position sense; K-L, Kellgren and Lawrence radiological assessment scale; KOOS, Knee Injury and Osteoarthritis Outcome Score; M, male; NRS, numeric rating scale; NSAID, non-steroidal anti-inflammatory drug; PEDro, Physiotherapy Evidence Database; PT, proprioceptive training; RCT, Randomized Controlled Trials; ROM, range of motion; RT, resistance training; SD, Standard deviation; ST, strength exercise; TE, traditional exercise; VAS, visual analog scale; WOMAC, Western Ontario and MCMaster Universities Osteoarthritis Index. 
TABLE 2 | PEDro scores of the included studies.

\begin{tabular}{|c|c|c|c|c|c|c|c|c|c|c|c|c|c|}
\hline Study & Year & $\begin{array}{l}\text { Eligibility } \\
\text { criteria }\end{array}$ & $\begin{array}{l}\text { Random } \\
\text { allocation }\end{array}$ & $\begin{array}{l}\text { Concealed } \\
\text { allocation }\end{array}$ & $\begin{array}{l}\text { Similar } \\
\text { baseline }\end{array}$ & $\begin{array}{l}\text { Blinding } \\
\text { subjects }\end{array}$ & $\begin{array}{l}\text { Blinding } \\
\text { therapists }\end{array}$ & $\begin{array}{l}\text { Blinding } \\
\text { assessors }\end{array}$ & $\begin{array}{l}\text { Dropout } \\
<15 \%\end{array}$ & $\begin{array}{l}\text { Intention to } \\
\text { treat }\end{array}$ & $\begin{array}{l}\text { Between- } \\
\text { group } \\
\text { statistics }\end{array}$ & $\begin{array}{l}\text { Point } \\
\text { measures }\end{array}$ & Total score \\
\hline Gomiero et al. (52) & 2018 & Yes & Yes & Yes & Yes & No & No & Yes & Yes & Yes & Yes & Yes & 8 \\
\hline Tsauo et al. (53) & 2008 & Yes & Yes & No & Yes & No & No & Yes & No & No & Yes & Yes & 5 \\
\hline Rogers et al. (40) & 2012 & Yes & Yes & Yes & Yes & Yes & No & No & No & No & Yes & Yes & 6 \\
\hline Sekir and Gür (60) & 2005 & Yes & Yes & No & Yes & No & No & No & Yes & Yes & Yes & Yes & 6 \\
\hline Rogers et al. (61) & 2011 & Yes & Yes & Yes & Yes & No & No & No & No & No & Yes & Yes & 5 \\
\hline Pazit et al. (58) & 2018 & Yes & Yes & Yes & Yes & No & No & Yes & Yes & Yes & Yes & Yes & 8 \\
\hline Takacs et al. (59) & 2017 & Yes & Yes & Yes & Yes & No & No & Yes & Yes & No & Yes & Yes & 7 \\
\hline Lin et al. (56) & 2007 & Yes & Yes & No & Yes & No & No & No & Yes & No & Yes & Yes & 5 \\
\hline Lin et al. (57) & 2009 & Yes & Yes & Yes & Yes & No & No & Yes & Yes & Yes & Yes & Yes & 8 \\
\hline Jahanjoo et al. (44) & 2019 & Yes & Yes & No & Yes & No & No & Yes & Yes & Yes & Yes & Yes & 7 \\
\hline $\begin{array}{l}\text { Chaipinyo and } \\
\text { Karoonsupcharoen } \\
\text { (62) }\end{array}$ & 2009 & Yes & Yes & Yes & Yes & No & No & No & Yes & No & Yes & Yes & 6 \\
\hline Fitzgerald et al. (41) & 2011 & Yes & Yes & Yes & Yes & No & No & Yes & No & Yes & Yes & Yes & 7 \\
\hline Duman et al. (69) & 2012 & Yes & Yes & No & Yes & No & No & No & Yes & Yes & Yes & Yes & 6 \\
\hline Diracoglu et al. (64) & 2005 & Yes & Yes & No & Yes & No & No & Yes & Yes & No & Yes & Yes & 6 \\
\hline Diracoglu et al. (66) & 2008 & Yes & Yes & No & Yes & No & No & Yes & Yes & No & Yes & Yes & 6 \\
\hline Ahmed (55) & 2011 & Yes & Yes & No & Yes & No & No & Yes & Yes & Yes & Yes & Yes & 7 \\
\hline Kumar et al. (54) & 2013 & Yes & Yes & No & Yes & No & No & No & Yes & Yes & Yes & Yes & 6 \\
\hline Elgendy et al. (65) & 2005 & Yes & Yes & No & Yes & No & No & No & Yes & Yes & Yes & Yes & 6 \\
\hline $\begin{array}{l}\text { Gohil and Shukla } \\
\text { (43) }\end{array}$ & 2020 & Yes & Yes & No & Yes & No & No & No & No & No & Yes & Yes & 4 \\
\hline Song et al. (45) & 2020 & Yes & Yes & No & Yes & No & No & No & No & No & Yes & Yes & 4 \\
\hline Kirthika et al. (39) & 2018 & Yes & Yes & No & Yes & No & Yes & Yes & Yes & Yes & Yes & Yes & 8 \\
\hline Mondam et al. (63) & 2012 & Yes & Yes & No & Yes & No & No & No & Yes & Yes & Yes & Yes & 6 \\
\hline Jan et al. (67) & 2009 & Yes & Yes & No & Yes & No & No & Yes & Yes & Yes & Yes & Yes & 7 \\
\hline Vamsidhar et al. (68) & 2017 & Yes & Yes & No & Yes & No & No & No & Yes & Yes & Yes & Yes & 6 \\
\hline
\end{tabular}

PEDro, Physiotherapy Evidence Database scale. 
95\%CI: $\left.[-0.58,0.23], I^{2}=0 \%, P=0.40\right)$. Additionally, the metaanalysis also showed no significant difference in relieving pain when proprioceptive training plus conventional physiotherapy compared against conventional physiotherapy ( $\mathrm{SMD}=-0.39$, 95\%CI: $[-0.99,0.22], I^{2}=68 \%, P=0.21$; Figure 2).

\section{Stiffness}

Seven studies $(40,44,53,58,61,66,69)$ used the WOMAC stiffness subscale to assess stiffness. When proprioceptive training compared to no intervention, only one study reported WOMAC stiffness score, and demonstrated that proprioceptive training effectively improved stiffness $(P<0.05)$. When proprioceptive training compared to other non-proprioceptive training, the subgroup analysis showed no statistically significant difference in improving stiffness (SMD $=-0.06,95 \% \mathrm{CI}$ : $[-0.78,0.65]$, $\left.I^{2}=0 \%, P=0.86\right)$. When proprioceptive training plus other non-proprioceptive training compared against other nonproprioceptive training, the meta-analysis revealed no significant difference (SMD $=-0.09,95 \% \mathrm{CI}$ : $[-0.69,0.50], I^{2}=43 \%, P=$ $0.76)$. Furthermore, the meta-analysis also showed no significant difference in improving stiffness when proprioceptive training plus conventional physiotherapy compared against conventional physiotherapy (SMD $=0.31,95 \%$ CI: $[-0.37,0.99], I^{2}=74 \%, P$ $=0.37$; Figure 3).

\section{Physical Function}

Eighteen studies (39-41, 43, 44, 52-54, 57-59, 61, 63, 64, 66-69) used the WOMAC physical function subscale and WOMAC total score to assess physical function. When proprioceptive training compared to no intervention, the subgroup analysis showed significant improvement in physical function (SMD $=-0.97$, 95\%CI: $\left.[-1.26,-0.67], I^{2}=0 \%, P<0.00001\right)$. Also, one study (40) showed statistically significant difference in WOMAC total score $(P<0.05)$. When proprioceptive training compared to other non-proprioceptive training, the meta-analysis showed no statistically significant difference in improving physical function $\left(\mathrm{SMD}=-0.03,95 \% \mathrm{CI}:[-0.75,0.69], I^{2}=3 \%, P=0.94\right)$. With respect to WOMAC total score, the meta-analysis also revealed no significant difference $(\mathrm{SMD}=-0.86,95 \% \mathrm{CI}$ : $[-2.68$, $0.97], I^{2}=93 \%, P=0.36$ ). When proprioceptive training plus other non-proprioceptive training compared against other nonproprioceptive training, the meta-analysis revealed significant improvement in physical function (SMD $=-0.34,95 \%$ CI: $\left.[-0.56,-0.12], I^{2}=0 \%, P=0.003\right)$. Similarly, the metaanalysis showed significant difference in WOMAC total score $\left(\mathrm{SMD}=-0.26,95 \% \mathrm{CI}:[-0.51,-0.01], I^{2}=0 \%, P=0.04\right)$. Furthermore, the meta-analysis showed no significant difference in improving physical function when proprioceptive training plus conventional physiotherapy compared against conventional physiotherapy (SMD $=0.01,95 \%$ CI: $[-0.57,0.60], I^{2}=77 \%, P$ $=0.97$ ). Similarly, the meta-analysis also showed no significant difference in WOMAC total score $(\mathrm{SMD}=-0.57,95 \% \mathrm{CI}$ : $[-1.69,0.54], I^{2}=93 \%, P=0.31$; Figures 4, 5).

\section{Secondary Outcomes \\ JPS}

Nine studies $(43,53-55,57,60,65,67,69)$ used the reposition error test to assess JPS. Subgroup analysis showed significant improvement in JPS for proprioceptive training compared to no intervention (SMD $=-1.73,95 \% \mathrm{CI}$ : $[-2.09,-1.37], I^{2}$ $=0 \%, P<0.00001)$. When proprioceptive training compared to other non-proprioceptive training, the meta-analysis showed significant difference in improving JPS (SMD $=-1.28,95 \% \mathrm{CI}$ : $\left.[-1.64,-0.92], I^{2}=0 \%, P<0.00001\right)$. When proprioceptive training plus other non-proprioceptive training compared against other non-proprioceptive training, the meta-analysis revealed statistically significant difference $(\mathrm{SMD}=-1.54$, 95\%CI: [-2.74, -0.34], $\left.I^{2}=79 \%, P=0.01\right)$. Additionally, the meta-analysis also showed significant improvement in JPS when proprioceptive training plus conventional physiotherapy compared against conventional physiotherapy (SMD $=-0.95$, $95 \% \mathrm{CI}$ : $[-1.73,-0.18], I^{2}=78 \%, P=0.02$; Figure 6).

\section{Muscle Strength}

Three studies $(56,62,67)$ assessed muscle strength by measuring knee flexion and extension torque. When proprioceptive training compared to no intervention, the subgroup analysis revealed that participants in the proprioceptive training group presented significantly increased knee flexion torque at the velocity of $60^{\circ} / \mathrm{s}$ $\left(\mathrm{SMD}=0.65,95 \% \mathrm{CI}:[0.29,1.01], I^{2}=0 \%, P=0.0004\right)$, but there was no significant difference at the velocity of 120 or $180^{\circ} / \mathrm{s}(P>0.05$; Table 3$)$. Likewise, there was also significant greater improvement of knee extension torque in participants of the proprioceptive training group at the velocity of $60 \% \mathrm{~s}$ $\left(\mathrm{SMD}=0.42,95 \% \mathrm{CI}:[0.07,0.78], I^{2}=0 \%, P=0.02\right)$, but no significant difference at the velocity of 120 or $180^{\circ} / \mathrm{s}$ $(P>0.05)$. When proprioceptive training compared against other non-proprioceptive training, the meta-analysis indicated that participants in the proprioceptive training group showed statistically significantly greater improvements in knee flexion torque at the velocity of $60^{\circ} / \mathrm{s}(\mathrm{SMD}=0.71,95 \% \mathrm{CI}$ : $[0.30,1.12]$, $I^{2}=0 \%, P=0.0008$; Table 4). Furthermore, Lin et al. (56) demonstrated that participants in the other non-proprioceptive training group presented significantly increased knee flexion torque at the velocity of 120 or $180^{\circ} / \mathrm{s}(P<0.05)$. Nevertheless, the meta-analysis showed no statistically significant difference between the groups $\left(\mathrm{SMD}=0.50,95 \% \mathrm{CI}:[-0.61,1.61], I^{2}=\right.$ $86 \%, P=0.38$ ) when knee extension torque was measured at the velocity of $60^{\circ} \%$ s. As in flexion, Lin et al. (56) indicated that participants in the other non-proprioceptive training group showed significantly higher knee flexion torque at the velocity of 120 or $180^{\circ} / \mathrm{s}(P<0.05)$.

\section{Mobility}

Two studies $(57,67)$ used the WST test to assess mobility. When proprioceptive training compared to no intervention, the subgroup analysis demonstrated that participants in the proprioceptive training group showed greater mobility when assessed for timed walk over ground $(\mathrm{SMD}=-0.57,95 \% \mathrm{CI}$ : $\left.[-0.90,-0.24], I^{2}=0 \%, P=0.0008\right)$, timed stair ascent and descent $\left(\mathrm{SMD}=-1.15,95 \% \mathrm{CI}\right.$ : $[-1.50,-0.79], I^{2}=$ $0 \%, P<0.00001$ ), and timed walk over spongy surface (SMD $=-1.66,95 \% \mathrm{CI}:[-2.05,-1.28], I^{2}=0 \%, P<0.00001$; Table 3). When proprioceptive training compared against other non-proprioceptive training, the meta-analysis revealed that participants in the proprioceptive training group presented 


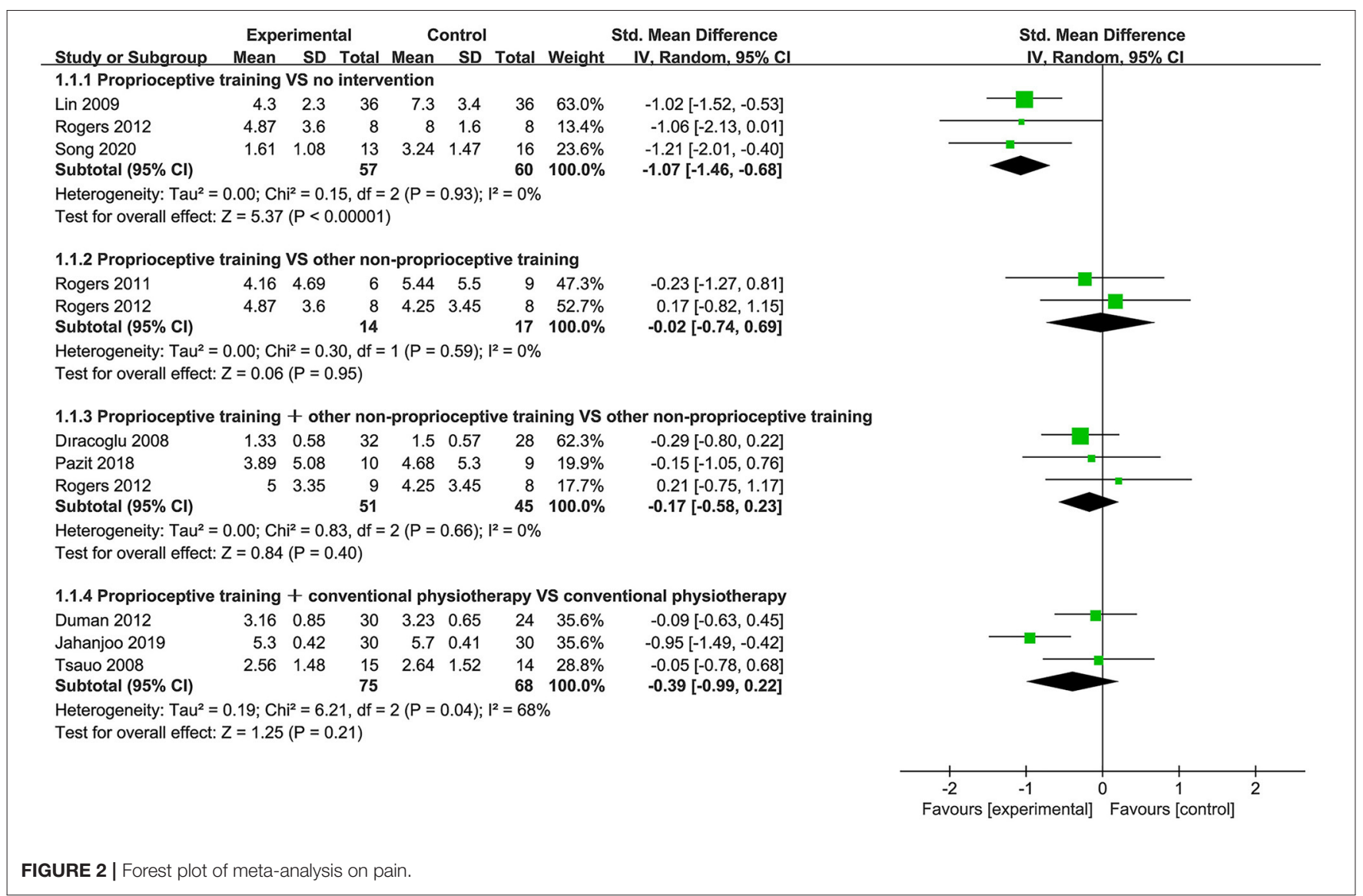

\begin{tabular}{|c|c|c|c|c|c|c|c|c|c|c|}
\hline \multirow[b]{2}{*}{ Study or Subgroup } & \multicolumn{3}{|c|}{ Experimental } & \multicolumn{2}{|c|}{ Control } & \multicolumn{3}{|c|}{ Std. Mean Difference } & \multirow{2}{*}{\multicolumn{2}{|c|}{$\begin{array}{l}\text { Std. Mean Difference } \\
\text { IV. Random, } 95 \% \mathrm{CI}\end{array}$}} \\
\hline & Mean & 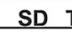 & Total & Mean & SD & Total & Weight & IV, Random, $95 \% \mathrm{CI}$ & & \\
\hline \multicolumn{11}{|c|}{ 1.2.1 Proprioceptive training VS other non-proprioceptive training } \\
\hline Rogers 2011 & 2 & 1.6 & 6 & 2.56 & 1.72 & 9 & $47.1 \%$ & $-0.31[-1.36,0.73]$ & & \\
\hline Rogers 2012 & 2.5 & 1.69 & 8 & 2.25 & 1.28 & 8 & $52.9 \%$ & $0.16[-0.82,1.14]$ & & \\
\hline Subtotal $(95 \% \mathrm{Cl})$ & & & 14 & & & & $100.0 \%$ & $-0.06[-0.78,0.65]$ & & \\
\hline \multicolumn{11}{|c|}{$\begin{array}{l}\text { Heterogeneity: } \text { Tau }^{2}=0.00 ; \mathrm{Chi}^{2}=0.42, \mathrm{df}=1(P=0.52) ; \mathrm{I}^{2}=0 \% \\
\text { Test for overall effect: } Z=0.18(P=0.86)\end{array}$} \\
\hline \multicolumn{11}{|c|}{ 1.2.2 Proprioceptive training + other non-proprioceptive training VS other non-proprioceptive training } \\
\hline Dıracoglu 2008 & 1.03 & 0.79 & 32 & 1.18 & 0.94 & 28 & $48.8 \%$ & $-0.17[-0.68,0.34]$ & & \\
\hline Pazit 2018 & 1.04 & 1.11 & 10 & 2.14 & 2.14 & 9 & $26.6 \%$ & $-0.63[-1.56,0.30]$ & & \\
\hline Rogers 2012 & 3.11 & 1.27 & 9 & 2.25 & 1.28 & 8 & $24.6 \%$ & $0.64[-0.34,1.62]$ & & \\
\hline Subtotal $(95 \% \mathrm{Cl})$ & & & 51 & & & & $100.0 \%$ & $-0.09[-0.69,0.50]$ & & \\
\hline \multicolumn{11}{|c|}{$\begin{array}{l}\text { Heterogeneity: } \text { Tau }^{2}=0.12 ; \mathrm{Ch}^{2}=3.48, \mathrm{df}=2(P=0.18) ; \mathrm{I}^{2}=43 \% \\
\text { Test for overall effect: } Z=0.31(P=0.76)\end{array}$} \\
\hline \multicolumn{11}{|c|}{ 1.2.3 Proprioceptive training + conventional physiotherapy VS conventional physiotherapy } \\
\hline Duman 2012 & 1.01 & 0.31 & 30 & 1.03 & 0.31 & 24 & $35.1 \%$ & $-0.06[-0.60,0.47]$ & & \\
\hline Jahanjoo 2019 & 1.7 & 0.32 & 30 & 1.4 & 0.31 & 30 & $35.2 \%$ & $0.94[0.40,1.48]$ & & \\
\hline Tsauo 2008 & 1.48 & 1.08 & 15 & 1.48 & 1.48 & 14 & $29.7 \%$ & $0.00[-0.73,0.73]$ & & \\
\hline Subtotal $(95 \% \mathrm{Cl})$ & & & 75 & & & 68 & $100.0 \%$ & $0.31[-0.37,0.99]$ & & \\
\hline \multicolumn{11}{|c|}{$\begin{array}{l}\text { Heterogeneity: } \text { Tau }^{2}=0.27 ; \mathrm{Chi}^{2}=7.83, \mathrm{df}=2(\mathrm{P}=0.02) ; \mathrm{I}^{2}=74 \% \\
\text { Test for overall effect: } Z=0.89(\mathrm{P}=0.37)\end{array}$} \\
\hline & & & & & & & & & $\begin{array}{ccc}-2 & -1 & 0 \\
\text { Favours [experimental] }\end{array}$ & $\begin{array}{cc}1 & 2 \\
\text { Favours [control] }\end{array}$ \\
\hline
\end{tabular}




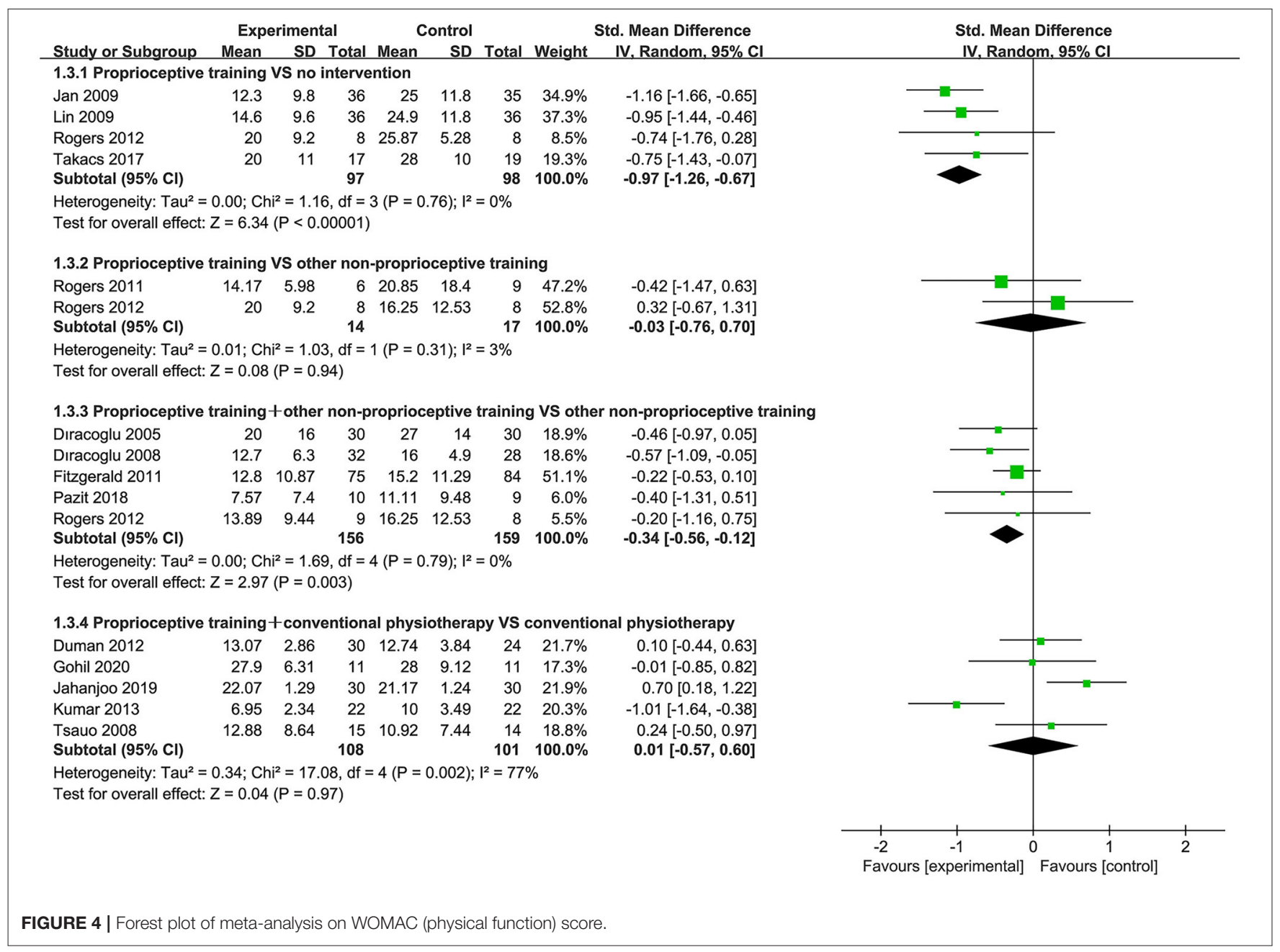

increased mobility when assessed for timed walk over spongy surface $\left(\mathrm{SMD}=-0.76,95 \% \mathrm{CI}\right.$ : $[-1.33,-0.18], I^{2}=0 \%, P=$ 0.01 ), but no statistically significant difference in timed walk over ground and timed stair ascent and descent $(P>0.05$; Table 4). Furthermore, four studies $(41,58,61,62)$ used the GUG test to assess mobility. Subgroup analysis showed no statistically significant difference between the groups when proprioceptive training compared against other non-proprioceptive training $\left(\mathrm{SMD}=-0.46,95 \% \mathrm{CI}:[-2.81,1.89], I^{2}=92 \%, P=\right.$ 0.70; Table 4). When proprioceptive training combined with other non-proprioceptive training compared against other nonproprioceptive training, the meta-analysis also revealed no significant difference in mobility $(\mathrm{SMD}=0.05,95 \% \mathrm{CI}$ : $[-0.55$, $0.65], I^{2}=46 \%, P=0.87$; Supplementary Figure S1).

\section{Knee ROM and Balance}

One study (45) used standard goniometric procedures (72) to measure knee ROM and showed significant improvement in knee ROM for proprioceptive training compared to no intervention $(P<0.05)$. In addition, when proprioceptive training plus other non-proprioceptive training compared against other nonproprioceptive training, Mondam et al. (63) used the Goniometer to measure knee ROM and demonstrated that there was statistically significantly greater knee ROM for the proprioceptive training plus other non-proprioceptive training group $(P<0.05)$. For balance, Gomiero et al. (52) assessed balance using the Tinetti balance scale and demonstrated that there was significant difference between the proprioceptive training group and the non-proprioceptive training group $(P<0.05)$.

\section{Adverse Events}

Only eight studies $(41,45,52,56,58,59,64,66)$ reported safety-related data, however some trials stated that no adverse events were reported $(41,64,66)$, no serious adverse events during the intervention occurred (58). In addition, from another four studies $(45,52,56,58)$ that provided data $(n=210)$, 12 participants $(5.7 \%)$ reported adverse events, including postexercise soreness, back pain, hip soreness, foot pain, and ankle injury.

\section{Sensitivity Analysis}

We used the leave-one-out method to conduct the sensitivity analyses for each of the evaluated outcomes (Supplementary Tables S3-S8). When proprioceptive training 


\begin{tabular}{|c|c|c|c|c|c|c|c|c|c|c|}
\hline \multirow[b]{2}{*}{ Study or Subgroup } & \multicolumn{3}{|c|}{ Experimental } & \multicolumn{3}{|c|}{ Control } & \multicolumn{2}{|c|}{ Std. Mean Difference } & \multirow{2}{*}{\multicolumn{2}{|c|}{$\begin{array}{r}\text { Std. Mean Difference } \\
\text { IV. Random, } 95 \% \mathrm{Cl}\end{array}$}} \\
\hline & Mean & SD & Total & Mean & & Total & Weight & IV, Random, $95 \% \mathrm{Cl}$ & & \\
\hline Gomiero 2018 & 30.6 & 18.33 & 32 & 29 & 16.54 & 32 & $35.3 \%$ & $0.09[-0.40,0.58]$ & & \\
\hline $\begin{array}{l}\text { Vamsidhar } 2017 \\
\text { Subtotal }(95 \% \mathrm{Cl})\end{array}$ & 23.4 & 1.99 & $\begin{array}{l}15 \\
55\end{array}$ & 29.67 & 1.99 & $\begin{array}{l}15 \\
55\end{array}$ & $\begin{array}{r}32.0 \% \\
100.0 \%\end{array}$ & $\begin{array}{l}-3.07[-4.16,-1.97] \\
-0.86[-2.68,0.97]\end{array}$ & & \\
\hline \multicolumn{11}{|c|}{$\begin{array}{l}\text { Heterogeneity: } \text { Tau }^{2}=2.39 ; \mathrm{Chi}^{2}=28.16, \mathrm{df}=2(P<0.00001) ;\left.\right|^{2}=93 \% \\
\text { Test for overall effect: } Z=0.92(P=0.36)\end{array}$} \\
\hline Dıracoglu 2008 & 22.1 & 9.6 & 32 & 26.1 & 8.9 & 28 & $23.2 \%$ & $-0.43[-0.94,0.09]$ & & \\
\hline Fitzgerald 2011 & 19 & 14.99 & 75 & 22 & 15.21 & 84 & $62.7 \%$ & $-0.20[-0.51,0.11]$ & & \\
\hline Pazit 2018 & 12.51 & 11.89 & 10 & 17.94 & 12.85 & 9 & $7.3 \%$ & $-0.42[-1.33,0.49]$ & & \\
\hline Rogers 2012 & 21.11 & 13.11 & 9 & 22.75 & 16.95 & 8 & $6.7 \%$ & $-0.10[-1.06,0.85]$ & & 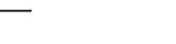 \\
\hline Subtotal $(95 \% \mathrm{Cl})$ & & & 126 & & & 129 & $100.0 \%$ & $-0.26[-0.51,-0.01]$ & & \\
\hline \multicolumn{11}{|c|}{$\begin{array}{l}\text { Heterogeneity: } \mathrm{Tau}^{2}=0.00 ; \mathrm{Chi}^{2}=0.77, \mathrm{df}=3(P=0.86) ; \mathrm{I}^{2}=0 \% \\
\text { Test for overall effect: } Z=2.06(P=0.04)\end{array}$} \\
\hline Mondam 2012 & 20.58 & 10.94 & $\begin{array}{r}25 \\
105\end{array}$ & 22.73 & 5.65 & 25 & $25.5 \%$ & $-0.24[-0.80,0.31]$ & & \\
\hline Subtotal (95\% Cl) & & & 105 & & & 99 & $100.0 \%$ & $-0.57[-1.69,0.54]$ & & \\
\hline \multicolumn{11}{|c|}{$\begin{array}{l}\text { Heterogeneity: } \text { Tau }^{2}=1.20 ; \mathrm{Chi}^{2}=41.68, \mathrm{df}=3(P<0.00001) ; I^{2}=93 \% \\
\text { Test for overall effect: } Z=1.01(P=0.31)\end{array}$} \\
\hline & & & & & & & & & Favours [experimental] & $\begin{array}{cr}2 & 4 \\
\text { Favours [control] }\end{array}$ \\
\hline
\end{tabular}

compared to other general non-proprioceptive training, the sensitivity analyses for WOMAC (total) score showed that the heterogeneity decreased to 0 after removing the study conducted by Vamsidhar et al. (68). When proprioceptive training plus conventional physiotherapy compared against conventional physiotherapy, the sensitivity analyses for pain and stiffness revealed that the heterogeneity decreased to 0 after removing Jahanjoo et al.'s study (44). And the sensitivity analyses for WOMAC (physical function) score showed that the removal of study conducted by Kumar et al. (54) significantly reduced the heterogeneity. The sensitivity analyses for WOMAC (total) score demonstrated that the removal of study conducted by Kirthika et al. (39) significantly reduced the heterogeneity. Furthermore, the sensitivity analyses for JPS showed the significant impact of proprioceptive training combined with conventional physiotherapy on JPS was highly affected by the study by Duman et al. (69). In general, these results suggested that these studies conducted by Vamsidhar et al. (68), Jahanjoo et al. (44), Kumar et al. (54), Kirthika et al. (39), or Duman et al. (69) could be the potential source of heterogeneity.

\section{Publication Bias}

No obvious asymmetry was found by the visual inspection of funnel plots (Figure 7). There was no evidence for publication bias on the WOMAC (physical function) score (Begg's test, $P=0.56$; Egger's regression, $P=0.95$ ) and the WOMAC (total) score (Begg's test, $P=0.28$; Egger's regression, $P=0.15)$.

\section{Quality of Evidence}

Based on the GRADE approach for evaluating quality of the evidence, the results showed that there is moderate evidence in pain, low to moderate evidence in stiffness, physical function, muscle strength and mobility, and very low to moderate evidence in JPS. The corresponding information are detailed in Supplementary Table S3.

\section{DISCUSSION}

Results of this meta-analysis indicated that compared to no intervention, proprioceptive training significantly improved pain, stiffness, physical function, JPS, muscle strength, mobility, and knee ROM in people with KOA. When compared to other non-proprioceptive training, proprioceptive training presented similar outcomes, only providing greater results in terms of JPS and mobility (timed walk over spongy surface). When proprioceptive training plus other non-proprioceptive training compared to other non-proprioceptive training, the two groups showed similar outcomes, but there was a greater improvement for JPS, physical function, and knee ROM in the proprioceptive training plus other non-proprioceptive training group. When proprioceptive training combined with conventional physiotherapy compared against conventional physiotherapy, the two groups demonstrated similar outcomes, but there was a significant improvement for JPS in the proprioceptive training combined with conventional physiotherapy group. In addition, although only eight studies reported safety-related data, considering the low rate of reported adverse events (5.7\%) 


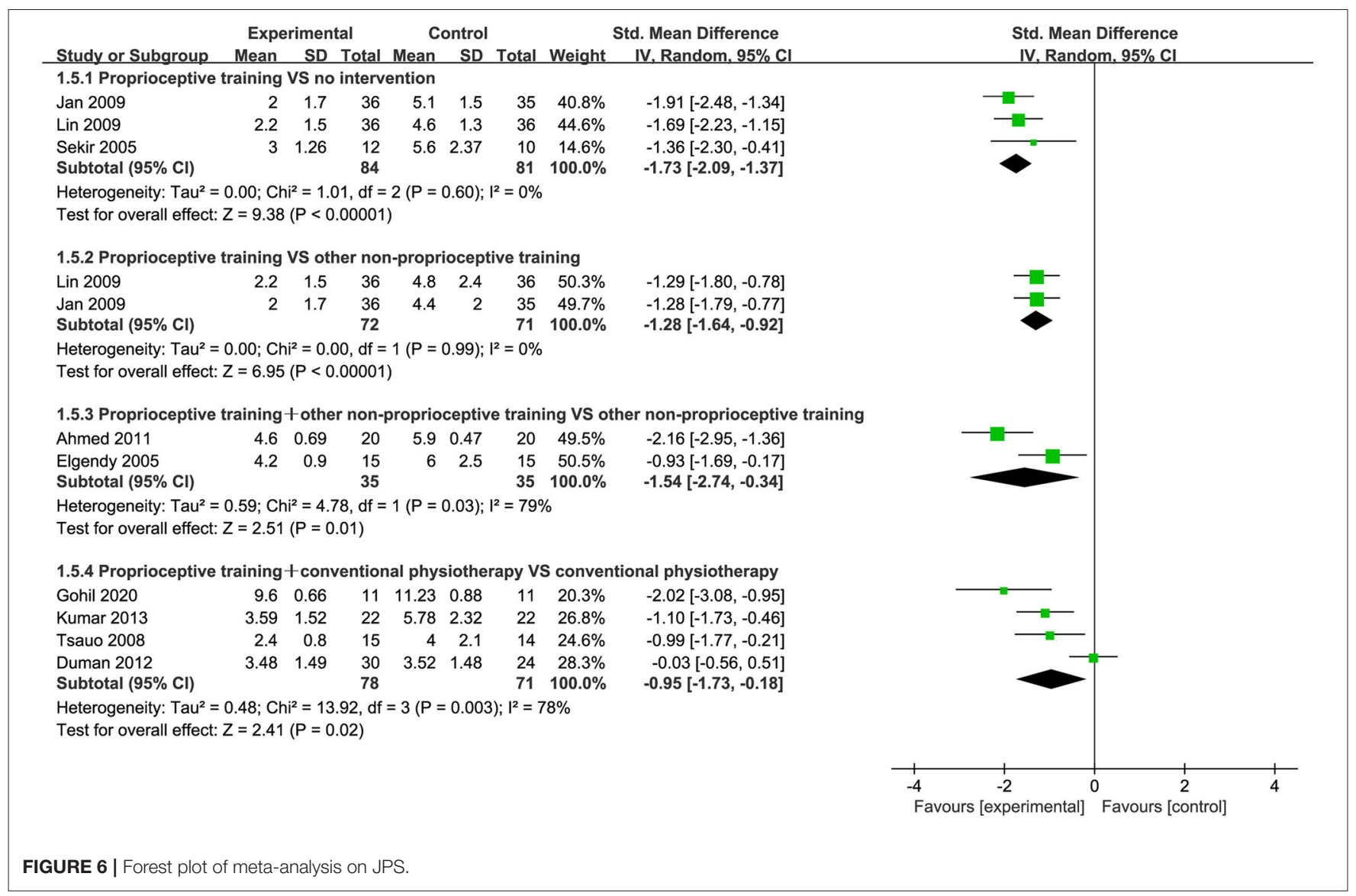

TABLE 3 | Meta-analysis results of muscle strength and mobility when proprioceptive training compared to no intervention.

\begin{tabular}{|c|c|c|c|c|c|}
\hline Outcomes & IG (sample size) & CG (sample size) & SMD $(95 \% \mathrm{Cl})$ & $P$-value & $I^{2}(\%)$ \\
\hline Knee flexion torque at $60^{\circ} / \mathrm{s}$ & 65 & 61 & $0.65(0.29,1.01)$ & 0.0004 & 0 \\
\hline Knee flexion torque at $120^{\circ} / \mathrm{s}$ & 65 & 61 & $0.32(-0.03,0.67)$ & 0.08 & 0 \\
\hline Knee flexion torque at $180^{\circ} / \mathrm{s}$ & 65 & 61 & $0.25(-0.10,0.60)$ & 0.17 & 0 \\
\hline Knee extension torque at $60 \%$ & 65 & 61 & $0.42(0.07,0.78)$ & 0.02 & 0 \\
\hline Knee extension torque at $120 \% \mathrm{~s}$ & 65 & 61 & $0.30(-0.05,0.65)$ & 0.09 & 0 \\
\hline Timed stair ascent and descent & 72 & 71 & $-1.15(-1.50,-0.79)$ & $<0.00001$ & 0 \\
\hline Timed walk over spongy surface & 72 & 71 & $-1.66(-2.05,-1.28)$ & $<0.00001$ & 0 \\
\hline
\end{tabular}

CG, control group; Cl, confidence interval; IG, intervention group; SMD, standard mean difference.

and about half of the reported adverse events $(n=5)$ were normal reactions after exercise, such as post-exercise soreness. Therefore, proprioceptive training could still be considered a relatively safe intervention for the treatment of KOA.

In this present study, the outcome measurements of JPS and walking speed over spongy surfaces were also specifically used to assess proprioception. When proprioceptive training compared to no intervention or other non-proprioceptive training, participants in proprioceptive training group showed greater improvement in the two outcomes. Similarly, when proprioceptive training plus other non-proprioceptive training compared against other non-proprioceptive training or proprioceptive training combined with conventional physiotherapy compared against conventional physiotherapy, participants in the groups that included proprioceptive training presented significantly improved JPS. This further demonstrated that the proprioceptive training could specifically improve knee proprioception in patients with $\mathrm{KOA}$, thereby indicating the effectiveness of this intervention.

We found that the measurements of the muscle strength/torque were inconsistent. Previous studies have indicated that the improvement of proprioception could 
TABLE 4 | Meta-analysis results of muscle strength and mobility when proprioceptive training compared to other non-proprioceptive training.

\begin{tabular}{|c|c|c|c|c|c|}
\hline Outcomes & IG (sample size) & CG (sample size) & SMD $(95 \% \mathrm{Cl})$ & $P$-value & $I^{2}(\%)$ \\
\hline Knee flexion torque at $60 \%$ s & 53 & 44 & $0.71(0.30,1.12)$ & 0.0008 & 0 \\
\hline Knee extension torque at $60 \%$ & 53 & 44 & $0.50(-0.61,1.61)$ & 0.38 & 86 \\
\hline Timed walk over ground & 72 & 71 & $0.06(-0.28,0.40)$ & 0.72 & 6 \\
\hline Timed stair ascent and descent & 72 & 71 & $0.35(-0.09,0.80)$ & 0.12 & 44 \\
\hline Timed walk over spongy surface & 72 & 71 & $-0.76(-1.33,-0.18)$ & 0.01 & 64 \\
\hline GUG test & 30 & 27 & $-0.46(-2.81,1.89)$ & 0.70 & 92 \\
\hline
\end{tabular}

CG, control group; Cl, confidence interval; GUG, Get up and go; IG, intervention group; SMD, standard mean difference.

promote the increase of muscle strength (73-75). The results were partially consistent with our findings. When proprioceptive training compared to other non-proprioceptive training, the intervention in the other non-proprioceptive training group was knee extension exercises. This may account for the finding that the greater improvement of knee extensor torque was observed in the other non-proprioceptive training group with respect to muscle strength of the knee extensors. In addition, we also found that there was a greater improvement for physical function and knee ROM in the proprioceptive training plus other non-proprioceptive training group. This may be due to the greater training intensity and frequency of the combined intervention, as well as the greater variety of training method.

\section{Comparison to Prior Reviews}

To our knowledge, this is the first systematic review and metaanalysis to evaluate the safety of proprioceptive training for KOA and the effects of combinations of proprioceptive training with other interventions in KOA. In recent years, only a few studies have systematically investigated the effect of proprioceptive training on knee proprioception and function in KOA. Smith et al. (35) conducted a study to determine the effectiveness of proprioceptive training for $\mathrm{KOA}$, the results indicated that significant improvement in functional outcomes (e.g., physical function, JPS, muscle strength, and mobility) for proprioceptive training compared to no intervention. When compared to other general non-proprioceptive training, proprioceptive training presented similar outcomes, only providing greater results in terms of JPS and mobility (timed walk over spongy surface). This is consistent with our review. In the most recent review (42), there is some evidence that proprioceptive training effectively improved pain and physical function in KOA, but stiffness and some other mobility measures (e.g., GUG) were unchanged after proprioceptive training. This is partially in line with our findings. The differences in the grouping of interventions may account for these inconsistent results.

\section{Limitations}

However, this present study had several following limitations. Firstly, methodologic limitations (e.g., inadequately concealed allocation, a small number of studies using therapists/subjects blinding or assessors blinding, high dropout rates, and inconsistent use of "intention-to-treat" analyses) could overestimate the overall effect size. Secondly, given the relatively small number of studies included in our review and the low number of participants per study, some results cannot be considered robust. Thirdly, since some of the included studies did not describe the race or age of the participants, we could not conduct a subgroup analysis based on race or age. Additionally, due to the relatively small number of included studies, subgroup analysis based on intervention characteristics (e.g., duration, dose or intensity) could not be carried out. Therefore, we could not determine the influence of these factors on the results. Fourthly, in our study, the components of the proprioceptive training programs in each study are different. We should perform the meta-analysis on each of the proprioceptive training separately, however, this is not possible given the few studies there are. These factors may also have a potential influence on our results. Furthermore, only two studies assessed outcomes after a year of follow-up. Accordingly, it is unable to determine the long-term effects of proprioceptive training in KOA.

\section{Implications for Further Research and Practice}

A larger and higher quality body of evidence is required before definite conclusions or recommendations could be made. Given that the main drawback of this review was the poor methodological quality of the included studies, future trials should use rigorous methodology to further ensure adequate concealed allocation, randomization, assessors blinding, and "intention-to-treat" analyses. Future researchers should improve the reporting in accordance with CONSORT guidelines (76). Future trials must also improve reporting of safety. Considering that this type of training may have potential therapeutic value, therefore it is necessary to further evaluate the optimal type of proprioceptive training. Additionally, the RCTs included in this review predominantly involved participants aged 50 years or over who presented early in their disease-stage. The majority of patients presented with moderate KOA (Kellgren-Lawrence grade II or III). Furthermore, the studies involving participants who had suffered knee joint trauma or surgery were also eliminated. Namely, this study only investigated patients with degenerative KOA. Given all this, the results of our study could only be applied to this KOA population. Therefore, it is still unclear whether the same clinical results would be obtained if proprioceptive 


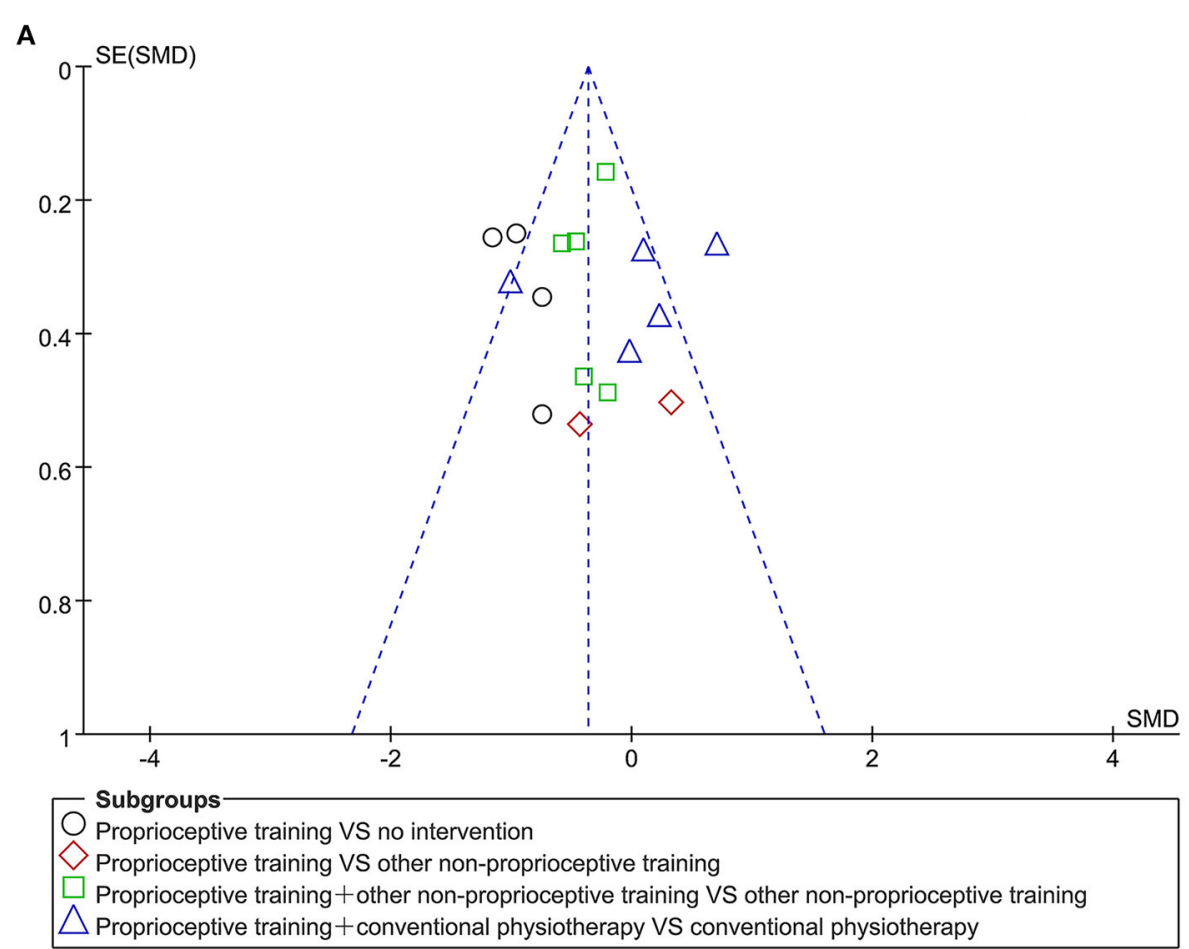

B

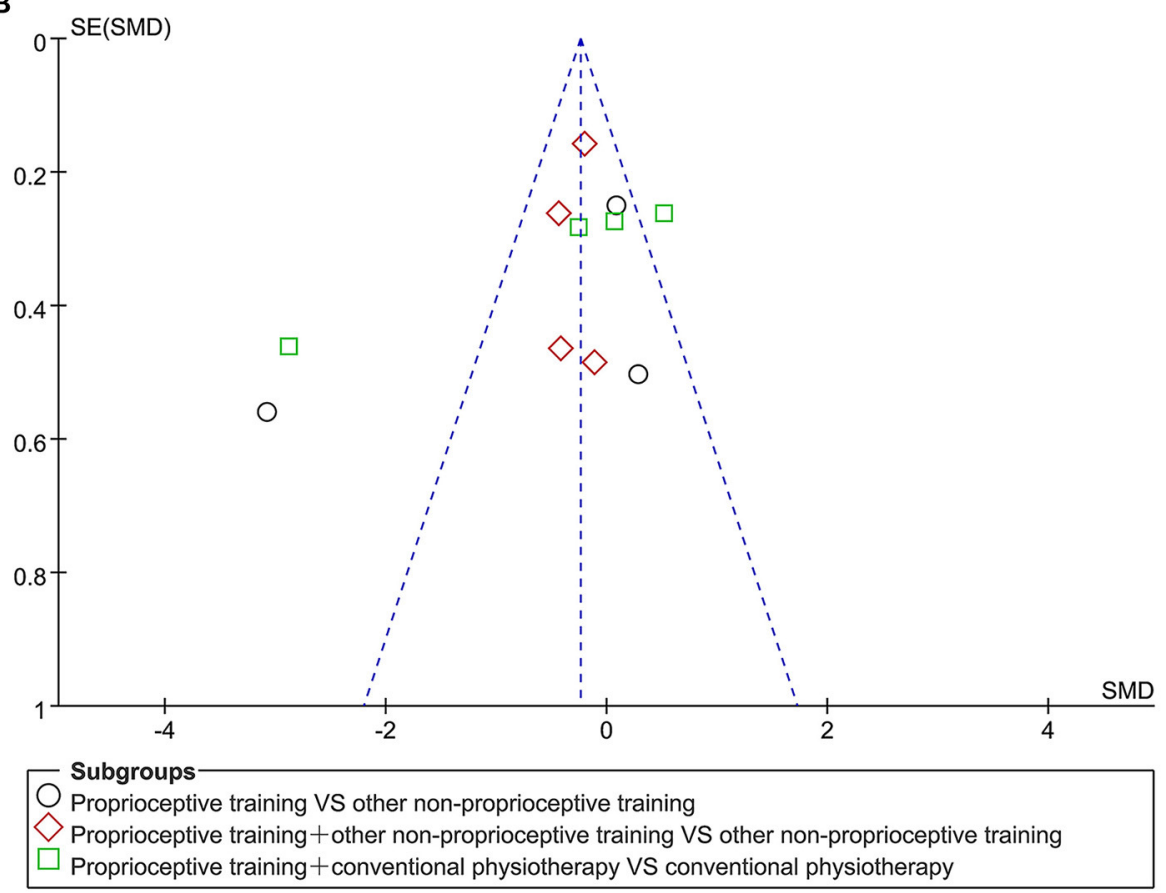

FIGURE 7 | Funnel plots for publication bias assessment on WOMAC (physical function) score (A) and WOMAC (total) score (B)

training was prescribed for older people or those with more advanced KOA or those with KOA due to joint trauma or surgery. Further studies are needed to evaluate the clinical applicability of this exercise regime in different populations with knee OA.

\section{CONCLUSIONS}

In conclusion, this present study indicated that proprioceptive training is safe and effective in treating KOA. There is some evidence that proprioceptive training combined with general 
non-proprioceptive training or conventional physiotherapy appears to be more effective and should be considered as part of the rehabilitation program. However, given that the majority of current studies investigated the short-term effect of these proprioceptive training program, more large-scale and well-designed studies with long-term follow up are needed to determine the long-term effects of these proprioceptive training regimes in $\mathrm{KOA}$.

\section{DATA AVAILABILITY STATEMENT}

The datasets presented in this study can be found in online repositories. The names of the repository/repositories and accession number(s) can be found in the article/Supplementary Material.

\section{AUTHOR CONTRIBUTIONS}

YW and XX came up with the initial study design. YW, ZW, $\mathrm{ZC}, \mathrm{XY}$, and JY reviewed all of the studies, extracted the data, analyzed and interpreted the data, and drafted the manuscript.

\section{REFERENCES}

1. Migliore A, Gigliucci G, Alekseeva L, Avasthi S, Bannuru RR, Chevalier X, et al. Treat-to-target strategy for knee osteoarthritis. International technical expert panel consensus and good clinical practice statements. Ther Adv Musculoskelet Dis. (2019) 11:1759720X19893800. doi: 10.1177/1759720X19893800

2. GBD 2017 Disease and Injury Incidence and Prevalence Collaborators. Global, regional, and national incidence, prevalence, and years lived with disability for 354 diseases and injuries for 195 countries and territories, 1990-2017: a systematic analysis for the Global Burden of Disease Study 2017. Lancet. (2018) 392:1789-858. doi: 10.1016/S0140-6736(18)32279-7

3. Neogi T. The epidemiology and impact of pain in osteoarthritis. Osteoarthr Cartil. (2013) 21:1145-53. doi: 10.1016/j.joca.2013.03.018

4. Reichenbach S, Felson DT, Hincapié CA, Heldner S, Bütikofer L, Lenz A, et al. Effect of biomechanical footwear on knee pain in people with knee osteoarthritis: the BIOTOK randomized clinical trial. JAMA. (2020) 323:1802-12. doi: 10.1001/jama.2020.3565

5. Cross M, Smith E, Hoy D, Nolte S, Ackerman I, Fransen M, et al. The global burden of hip and knee osteoarthritis: estimates from the global burden of disease 2010 study. Ann Rheum Dis. (2014) 73:132330. doi: 10.1136/annrheumdis-2013-204763

6. Abbott JH, Usiskin IM, Wilson R, Hansen P, Losina E. The quality-of-life burden of knee osteoarthritis in New Zealand adults: a model-based evaluation. PLoS ONE. (2017) 12:e0185676. doi: 10.1371/journal.pone.0185676

7. Sharma L, Dunlop DD, Cahue S, Song J, Hayes KW. Quadriceps strength and osteoarthritis progression in malaligned and lax knees. Ann Intern Med. (2003) 138:613-9. doi: 10.7326/0003-4819-138-8-200304150-00006

8. Sharma L. Osteoarthritis of the knee. N Engl J Med. (2021) 384:519. doi: 10.1056/NEJMcp1903768

9. Temple-Wong MM, Ren S, Quach P, Hansen BC, Chen AC, Hasegawa A, et al. Hyaluronan concentration and size distribution in human knee synovial fluid: variations with age and cartilage degeneration. Arthritis Res Ther. (2016) 18:18. doi: 10.1186/s13075-016-0922-4

10. Arden N, Nevitt MC. Osteoarthritis: epidemiology. Best Pract Res Clin Rheumatol. (2006) 20:3-25. doi: 10.1016/j.berh.2005.09.007

11. Hochberg MC, Altman RD, April KT, Benkhalti M, Guyatt G, McGowan J, et al. American College of Rheumatology 2012 recommendations
YW, ZW, YG, JW, and GC were involved in quality assessment. $\mathrm{PZ}, \mathrm{FX}, \mathrm{WC}$, and $\mathrm{ZY}$ checked the data extraction and analysis for accuracy. XX critically revised the manuscript for important intellectual content. All authors have reviewed and approved the final version of the manuscript.

\section{FUNDING}

This work was supported by Science and Technology Innovation Strategy Special Fund of Guangdong Province (2021B1111610007); Science and Technology Plan Project of Guangdong Province (2019A141401008); Soft Science Research Project of Guangdong Province (No. 2018B020207009); and Natural Science Foundation of Guangdong Province (2021A1515011545).

\section{SUPPLEMENTARY MATERIAL}

The Supplementary Material for this article can be found online at: https://www.frontiersin.org/articles/10.3389/fmed. 2021.699921/full\#supplementary-material for the use of nonpharmacologic and pharmacologic therapies in osteoarthritis of the hand, hip, and knee. Arthritis Care Res. (2012) 64:465-74. doi: 10.1002/acr.21596

12. Clegg DO, Reda DJ, Harris CL, Klein MA, O'Dell JR, Hooper $M M$, et al. Glucosamine, chondroitin sulfate, and the two in combination for painful knee osteoarthritis. N Engl J Med. (2006) 354:795-808. doi: 10.1056/NEJMoa052771

13. Fernandes L, Hagen KB, Bijlsma JW, Andreassen O, Christensen P, Conaghan $\mathrm{PG}$, et al. EULAR recommendations for the non-pharmacological core management of hip and knee osteoarthritis. Ann Rheum Dis. (2013) 72:112535. doi: 10.1136/annrheumdis-2012-202745

14. McAlindon TE, Bannuru RR, Sullivan MC, Arden NK, Berenbaum F, Bierma-Zeinstra SM, et al. OARSI guidelines for the non-surgical management of knee osteoarthritis. Osteoarthr Cartil. (2014) 22:36388. doi: $10.1016 /$ j.joca.2014.01.003

15. Birmingham TB, Kramer JF, Kirkley A, Inglis JT, Spaulding SJ, Vandervoort AA. Knee bracing for medial compartment osteoarthritis: effects on proprioception and postural control. Rheumatology. (2001) 40:285-9. doi: 10.1093/rheumatology/40.3.285

16. Nor Azlin MN, Lyn KS. Effects of passive joint mobilization on patients with knee osteoarthritis. Sains Malays. (2011) 40:1461-5.

17. Zhang W, Moskowitz RW, Nuki G, Abramson S, Altman RD, Arden N, et al. OARSI recommendations for the management of hip and knee osteoarthritis, part II: OARSI evidence-based, expert consensus guidelines. Osteoarthr Cartil. (2008) 16:137-62. doi: 10.1016/j.joca.2007.12.013

18. Martel-Pelletier J, Barr AJ, Cicuttini FM, Conaghan PG, Cooper C, Goldring MB, et al. Osteoarthritis. Nat Rev Dis Primers. (2016) 2:16072. doi: 10.1038/nrdp.2016.72

19. Bennell KL, Hunter DJ, Hinman RS. Management of osteoarthritis of the knee. BMJ. (2012) 345:e4934. doi: 10.1136/bmj.e4934

20. Vincent KR, Vincent HK. Concentric and eccentric resistance training comparison on physical function and functional pain outcomes in knee osteoarthritis: a randomized controlled trial. Am J Phys Med Rehabil. (2020) 99:932-40. doi: 10.1097/PHM.0000000000001450

21. Huang CC, Wang HH, Chen KC, Yang KJ, Chang LY, Shiang TY, et al. Effects of a dynamic combined training on impulse response for middle-aged and elderly patients with osteoporosis and knee osteoarthritis: a randomized control trial. Aging Clin Exp Res. (2021) 33:115-23. doi: 10.1007/s40520-020-01508-0 
22. Silverwood V, Blagojevic-Bucknall M, Jinks C, Jordan JL, Protheroe J, Jordan KP. Current evidence on risk factors for knee osteoarthritis in older adults: a systematic review and meta-analysis. Osteoarthr Cartil. (2015) 23:50715. doi: 10.1016/j.joca.2014.11.019

23. Glyn-Jones S, Palmer AJ, Agricola R, Price AJ, Vincent TL, Weinans $\mathrm{H}$, et al. Osteoarthritis. Lancet. (2015) 386:37687. doi: 10.1016/S0140-6736(14)60802-3

24. Krishnasamy P, Hall M, Robbins SR. The role of skeletal muscle in the pathophysiology and management of knee osteoarthritis. Rheumatology. (2018) 57:iv22-33. doi: 10.1093/rheumatology/kex515

25. Muollo V, Rossi AP, Zignoli A, Teso M, Milanese C, Cavedon V, et al. Full characterisation of knee extensors' function in ageing: effect of sex and obesity. Int J Obes. (2021) 45:895-905. doi: 10.1038/s41366-021-00755-Z

26. Muollo V, Zignoli A, Ghiotto L, Milanese C, Zamboni M, Schena F, et al. Knee flexor and extensor torque ratio in elderly men and women with and without obesity: a cross-sectional study. Aging Clin Exp Res. (2021) doi: 10.1007/s40520-021-01884-1

27. Felson DT, Gross KD, Nevitt MC, Yang M, Lane NE, Torner JC, et al. The effects of impaired joint position sense on the development and progression of pain and structural damage in knee osteoarthritis. Arthritis Rheum. (2009) 61:1070-6. doi: 10.1002/art.24606

28. Lund H, Juul-Kristensen B, Hansen K, Christensen R, Christensen H, Danneskiold-Samsoe B, et al. Movement detection impaired in patients with knee osteoarthritis compared to healthy controls: a cross-sectional casecontrol study. J Musculoskelet Neuronal Interact. (2008) 8:391-400.

29. Knoop J, Steultjens MP, van der Leeden M, van der Esch M, Thorstensson CA, Roorda LD, et al. Proprioception in knee osteoarthritis: a narrative review. Osteoarthr Cartil. (2011) 19:381-8. doi: 10.1016/j.joca.2011. 01.003

30. Hadamus A, Białoszewski D. Objective assessment of knee proprioception and sensorimotor function in patients with primary gonarthrosis before and after knee replacement. Ortop Traumatol Rehabil. (2017) 19:40314. doi: 10.5604/01.3001.0010.5820

31. Bayramoglu M, Toprak R, Sozay S. Effects of osteoarthritis and fatigue on proprioception of the knee joint. Arch Phys Med Rehabil. (2007) 88:34650. doi: 10.1016/j.apmr.2006.12.024

32. Stillman BC. Making sense of proprioception: the meaning of proprioception, kinaesthesia and related terms. Physiotherapy. (2002) 88:667-76. doi: 10.1016/S0031-9406(05)60109-5

33. Sharma L. Proprioceptive impairment in knee osteoarthritis. Rheum Dis Clin North Am. (1999) 25:299-314, vi. doi: 10.1016/s0889-857x(05)70069-7

34. Olsson L, Lund H, Henriksen M, Rogind H, Bliddal H, DanneskioldSamse B. Test-retest reliability of a knee joint position sense measurement method in sitting and prone position. Adv Physiother. (2004) 6:3747. doi: 10.1080/14038190310009894

35. Smith TO, King JJ, Hing CB. The effectiveness of proprioceptive-based exercise for osteoarthritis of the knee: a systematic review and meta-analysis. Rheumatol Int. (2012) 32:3339-51. doi: 10.1007/s00296-012-2480-7

36. Van Ginckel A, Hall M, Dobson F, Calders P. Effects of long-term exercise therapy on knee joint structure in people with knee osteoarthritis: a systematic review and meta-analysis. Semin Arthritis Rheum. (2019) 48:9419. doi: 10.1016/j.semarthrit.2018.10.014

37. van Dijk GM, Dekker J, Veenhof C, van den Ende CH, Carpa Study Group. Course of functional status and pain in osteoarthritis of the hip or knee: a systematic review of the literature. Arthritis Rheum. (2006) 55:77985. doi: 10.1002/art.22244

38. Aman JE, Elangovan N, Yeh IL, Konczak J. The effectiveness of proprioceptive training for improving motor function: a systematic review. Front Hum Neurosci. (2015) 8:1075. doi: 10.3389/fnhum.2014.01075

39. Kirthika V, Sudhakar S, Padmanabhan K, Ramachandran S, Kumar M. Efficacy of combined proprioceptive exercises and conventional physiotherapy in patients with knee osteoarthritis: a double-blinded two-group pretest-posttest design. Ortop Traumatol Rehabil. (2018) 10:94. doi: 10.4103/jotr.jotr_40_17

40. Rogers MW, Tamulevicius N, Semple SJ, Krkeljas Z. Efficacy of homebased kinesthesia, balance \& agility exercise training among persons with symptomatic knee osteoarthritis. J Sports Sci Med. (2012) 11:751-8.

41. Fitzgerald GK, Piva SR, Gil AB, Wisniewski SR, Oddis CV, Irrgang JJ. Agility and perturbation training techniques in exercise therapy for reducing pain and improving function in people with knee osteoarthritis: a randomized clinical trial. Phys Ther. (2011) 91:452-69. doi: 10.2522/ptj.20100188

42. Jeong HS, Lee SC, Jee H, Song JB, Chang HS, Lee SY. Proprioceptive training and outcomes of patients with knee osteoarthritis: a metaanalysis of randomized controlled trials. J Athl Train. (2019) 54:41828. doi: 10.4085/1062-6050-329-17

43. Gohil M, Shukla Y. Effect of proprioceptive training using biofeedback (equiboard) on pain, function and proprioception in osteoarthritic knee-an interventional study. Population. (2020) 2:3.

44. Jahanjoo F, Eftekharsadat B, Bihamta A, Babaei-Ghazani A. Efficacy of balance training in combination with physical therapy in rehabilitation of knee osteoarthritis: a randomized clinical trial. Crescent J Med Biol Sci. (2019) 6:225-334

45. Song Q, Shen P, Mao M, Sun W, Zhang C, Li L. Proprioceptive neuromuscular facilitation improves pain and descending mechanics among elderly with knee osteoarthritis. Scand J Med Sci Sports. (2020) 30:165563. doi: 10.1111/sms.13709

46. Moher D, Liberati A, Tetzlaff J, Altman DG, PRISMA Group. Preferred reporting items for systematic reviews and meta-analyses: the PRISMA statement. PLoS Med. (2009) 6:e1000097. doi: 10.1371/journal.pmed.1000097

47. Guyatt GH, Oxman AD, Schünemann HJ, Tugwell P, Knottnerus A. GRADE guidelines: a new series of articles in the Journal of Clinical Epidemiology. $J$ Clin Epidemiol. (2011) 64:380-2. doi: 10.1016/j.jclinepi.2010.09.011

48. Macedo LG, Elkins MR, Maher CG, Moseley AM, Herbert RD, Sherrington C. There was evidence of convergent and construct validity of Physiotherapy Evidence Database quality scale for physiotherapy trials. J Clin Epidemiol. (2010) 63:920-5. doi: 10.1016/j.jclinepi.2009.10.005

49. Maher CG, Sherrington C, Herbert RD, Moseley AM, Elkins M. Reliability of the PEDro scale for rating quality of randomized controlled trials. Phys Ther. (2003) 83:713-21

50. Higgins JPT, Thomas J, Chandler J, Cumpston M, Li T, Page MJ, et al. Cochrane Handbook for Systematic Reviews of Interventions Version 6.2 (Updated February 2021). Cochrane (2021). Available online at: www.training.cochrane.org/handbook (accessed October 13, 2021).

51. Higgins JP, Thompson SG. Quantifying heterogeneity in a meta-analysis. Stat Med. (2002) 21:1539-58. doi: 10.1002/sim.1186

52. Gomiero AB, Kayo A, Abraão M, Peccin MS, Grande AJ, Trevisani VF. Sensory-motor training versus resistance training among patients with knee osteoarthritis: randomized single-blind controlled trial. São Paulo Med J. (2018) 136:44-50. doi: 10.1590/1516-3180.2017.0174100917

53. Tsauo JY, Cheng PF, Yang RS. The effects of sensorimotor training on knee proprioception and function for patients with knee osteoarthritis: a preliminary report. Clin Rehabil. (2008) 22:448-57. doi: 10.1177/0269215507084597

54. Kumar S, Kumar A, Kumar R. Proprioceptive training as an adjunct in osteoarthritis of knee. J Musculoskelet Res. (2013) 16:1350002. doi: 10.1142/S0218957713500024

55. Ahmed AF. Effect of sensorimotor training on balance in elderly patients with knee osteoarthritis. J Adv Res. (2011) 2:305-11. doi: 10.1016/j.jare.2011.02.001

56. Lin DH, Lin YF, Chai HM, Han YC, Jan MH. Comparison of proprioceptive functions between computerized proprioception facilitation exercise and closed kinetic chain exercise in patients with knee osteoarthritis. Clin Rheumatol. (2007) 26:520-8. doi: 10.1007/s10067-006-0324-0

57. Lin $\mathrm{DH}$, Lin $\mathrm{CH}$, Lin YF, Jan MH. Efficacy of 2 non-weight-bearing interventions, proprioception training versus strength training, for patients with knee osteoarthritis: a randomized clinical trial. J Orthop Sports Phys Ther. (2009) 39:450-7. doi: 10.2519/jospt.2009.2923

58. Pazit L, Jeremy D, Nancy B, Michael B, George E, Hill KD. Safety and feasibility of high speed resistance training with and without balance exercises for knee osteoarthritis: a pilot randomised controlled trial. Phys Ther Sport. (2018) 34:154-63. doi: 10.1016/j.ptsp.2018.10.001

59. Takacs J, Krowchuk NM, Garland SJ, Carpenter MG, Hunt MA. Dynamic balance training improves physical function in individuals with knee osteoarthritis: a pilot randomized controlled trial. Arch Phys Med Rehabil. (2017) 98:1586-93. doi: 10.1016/j.apmr.2017.01.029

60. Sekir U, Gür H. A multi-station proprioceptive exercise program in patients with bilateral knee osteoarthrosis: functional capacity, pain and sensorimotor function. A randomized controlled trial. J Sports Sci Med. (2005) 4:590-603. 
61. Rogers MW, Tamulevicius N, Coetsee MF, Curry BF, Semple SJ. Knee osteoarthritis and the efficacy of kinesthesia, balance \& agility exercise training: a pilot study. Int J Exerc Sci. (2011) 4:124-32.

62. Chaipinyo K, Karoonsupcharoen O. No difference between home-based strength training and home-based balance training on pain in patients with knee osteoarthritis: a randomised trial. Aust J Physiother. (2009) 55:2530. doi: 10.1016/s0004-9514(09)70057-1

63. Mondam S, Babu S, Kumar R, Prakash J. A comparative study of proprioceptive exercises versus conventional training program on osteoarthritis of knee. Res J Recent Sci. (2012) 1:31-5.

64. Diracoglu D, Aydin R, Baskent A, Celik A. Effects of kinesthesia and balance exercises in knee osteoarthritis. J Clin Rheumatol. (2005) 11:30310. doi: 10.1097/01.rhu.0000191213.37853.3d

65. Elgendy M, Amin F, Baraka H, Khaled O. Influence of proprioceptive training on knee function in patients with knee osteoarthritis. Bull Fac Ph Th Cairo Univ. (2005) 10(2).

66. Diracoglu D, Baskent A, Celik A, Issever H, Aydin R. Long-term effects of kinesthesia/balance and strengthening exercises on patients with knee osteoarthritis: a one-year follow-up study. J Back Musculoskelet Rehabil. (2008) 21:253-62. doi: 10.3233/BMR-2008-21406

67. Jan $\mathrm{MH}$, Lin $\mathrm{CH}$, Lin YF, Lin JJ, Lin DH. Effects of weight-bearing versus nonweight-bearing exercise on function, walking speed, and position sense in participants with knee osteoarthritis: a randomized controlled trial. Arch Phys Med Rehabil. (2009) 90:897-904. doi: 10.1016/j.apmr.2008. 11.018

68. Vamsidhar N, Reddy RSK, Thiruppathi A, Ravindran B, Sivakumar B. A study to find the effectiveness of proprioceptive training and strengthening exercises of osteoarthritis of knee. J Med Sci Clin Res. (2017) 5:233525. doi: 10.18535/jmscr/v5i6.93

69. Duman I, Taskaynatan MA, Mohur H, Tan AK. Assessment of the impact of proprioceptive exercises on balance and proprioception in patients with advanced knee osteoarthritis. Rheumatol Int. (2012) 32:37938. doi: 10.1007/s00296-011-2272-5

70. Kellgren JH, Lawrence JS. Radiological assessment of osteoarthrosis. Ann Rheum Dis. (1957) 16:494-502. doi: 10.1136/ard.16.4.494

71. Altman R, Asch E, Bloch D, Bole G, Borenstein D, Brandt K, et al. Development of criteria for the classification and reporting of osteoarthritis. Classification of osteoarthritis of the knee. Diagnostic and Therapeutic
Criteria Committee of the American Rheumatism Association. Arthritis Rheum. (1986) 29:1039-49. doi: 10.1002/art.1780290816

72. Hindle KB, Whitcomb TJ, Briggs WO, Hong J. Proprioceptive neuromuscular facilitation (PNF): its mechanisms and effects on range of motion and muscular function. J Hum Kinet. (2012) 31:105-13. doi: 10.2478/v10078-012-0011-y

73. Hurley MV, Scott DL. Improvements in quadriceps sensorimotor function and disability of patients with knee osteoarthritis following a clinically practicable exercise regime. Br J Rheumatol. (1998) 37:1181-7. doi: 10.1093/rheumatology/37.11.1181

74. Bouët V, Gahéry Y. Muscular exercise improves knee position sense in humans. Neurosci Lett. (2000) 289:1436. doi: 10.1016/s0304-3940(00)01297-0

75. Hewett TE, Paterno MV, Myer GD. Strategies for enhancing proprioception and neuromuscular control of the knee. Clin Orthop Relat Res. (2002) 402:7694. doi: 10.1097/00003086-200209000-00008

76. Schulz KF, Altman DG, Moher D, CONSORT Group. CONSORT 2010 statement: updated guidelines for reporting parallel group randomised trials. BMJ. (2010) 340:c332. doi: 10.1136/bmj.c332

Conflict of Interest: The authors declare that the research was conducted in the absence of any commercial or financial relationships that could be construed as a potential conflict of interest.

Publisher's Note: All claims expressed in this article are solely those of the authors and do not necessarily represent those of their affiliated organizations, or those of the publisher, the editors and the reviewers. Any product that may be evaluated in this article, or claim that may be made by its manufacturer, is not guaranteed or endorsed by the publisher.

Copyright (c) 2021 Wang, Wu, Chen, Ye, Chen, Yang, Zhang, Xie, Guan, Wu, Chen, $Y e$ and $X u$. This is an open-access article distributed under the terms of the Creative Commons Attribution License (CC BY). The use, distribution or reproduction in other forums is permitted, provided the original author(s) and the copyright owner(s) are credited and that the original publication in this journal is cited, in accordance with accepted academic practice. No use, distribution or reproduction is permitted which does not comply with these terms. 